\title{
Rothe time-discretization method for a nonlinear parabolic problem in Orlicz-Sobolev spaces
}

\section{Lakbir Essafi $^{1} \mathbb{D} \cdot$ Said Hadj Nassar ${ }^{1} \cdot$ Mohamed Rhoudaf $^{2}$}

Received: 25 August 2017 / Accepted: 11 January 2019 / Published online: 25 January 2019

(c) The Author(s) 2019

\section{Abstract}

In this paper, we prove the existence and uniqueness of entropy solutions for the following equations in Orlicz spaces:

$$
\left\{\begin{array}{c}
\left.\frac{\partial u}{\partial t}-\operatorname{div}(a(x, \nabla u(x, t)))+\beta(u)=f \text { in } Q_{T}=\right] 0 . T[\times \Omega \\
\left.u=0 \text { on } \Sigma_{T}=\right] 0 . T[\times \partial \Omega \\
u(0, .)=0 \text { in } \Omega,
\end{array}\right.
$$

where $f$ is an element of $L^{1}\left(Q_{T}\right)$, the term $-\operatorname{div}(a(x, \nabla u(x, t)))$ is a Leray-Lions operator on $W_{0}^{1, x} L_{M}(\Omega)$, with $M\left(\right.$.) does not satisfy the $\Delta_{2}$ condition and $\beta$ is a continuous non decreasing real function defined on $\mathbb{R}$ with $\beta(0)=0$. The investigation is made by approximation of the Rothe method which is based on a semi-discretization of the given problem with respect to the time variable.

Keywords Rothe method - Orlicz spaces · Truncation · Parabolic · Entropy solution · Existence $\cdot$ Stability $\cdot$ Uniqueness

Mathematics Subject Classification $35 \mathrm{~K} 55 \cdot 35 \mathrm{~J} 60$

Lakbir Essafi

essafi20@gmail.com

Said Hadj Nassar

hnasser@fstt.ac.ma

Mohamed Rhoudaf

rhoudafmohamed@gmail.com

1 Département de Mathématiques, Faculté des Sciences et Techniques, Université Abdelmalek Essaadi, Tangier, Morocco

2 Département Mathématiques, Faculté des Sciences, Université Moulay Ismail, Meknes, Morocco 


\section{Introduction}

The study of variational problems where the operator associated to a PDEs satisfies the non-polynomial growth conditions instead of having the usual $p$-structure arouses much interest with the development of applications to electro-rheological fluids as an important class of non-Newtonian fluids (sometimes referred to as smart fluids). The electro-rheological fluids are characterized by their ability to drastically change the mechanical properties under the influence of an external electromagnetic field. A mathematical model of electro-rheological fluids was proposed by Rajagopal and Ruzicka (we refer to [31,33] for more details). Another important application is related to image processing [29] where this kind of the diffusion operator is used to underline the borders of the distorted image and to eliminate the noise.

Let $\Omega$ be a bounded open subset of $\mathbb{R}^{d}, d \geq 2$, with segment property and $A(u)=-\operatorname{div}(a(x, \nabla u(x, t)))$ is a Leray-Lions operator defined on $W_{0}^{1, x} L_{M}\left(Q_{T}\right)$, where $\mathrm{M}$ is an $\mathrm{N}$-function without assuming a $\Delta_{2}$-condition on $\mathrm{M}$.

We consider the following nonlinear parabolic problem:

$$
\left\{\begin{array}{c}
\left.\frac{\partial u}{\partial t}-\operatorname{div}(a(x, \nabla u(x, t)))+\beta(u)=f \text { in } Q_{T}=\right] 0 . T[\times \Omega \\
\left.u=0 \text { on } \Sigma_{T}=\right] 0 . T[\times \partial \Omega \\
u(0, .)=0 \text { in } \Omega,
\end{array}\right.
$$

where the data $f \in L^{1}\left(Q_{T}\right)$ and $\beta$ is taken as continuous non decreasing real function everywhere defined on $\mathbb{R}$ with $\beta(0)=0$.

Under these assumptions, the above problem does not admit, in general, a weak solution since the fields $a(x, \nabla u(x, t))$ do not belong to $\left(L_{l o c}^{1}\left(Q_{T}\right)\right)^{d}$. To overcome this difficulty we use the framework of entropy solutions was introduced by Benilan and al. [4] for the nonlinear elliptic problems. The concept of entropy solution in the case parabolic problem was obtained in [28], At the same the equivalent notion of renormalized solution has been introduced by Lions and Di Perna [6] for the study of Boltzmann equation, this notion was then adapted to elliptic vesion by Boccardo, J.-L. Diaz, D. Giachetti, F. Murat [5].

Note that in the case of classical Sobolev spaces $W^{1, p}$ J. Droniou and A. Prignet in [7] demonstrated the equivalence between entropy and renormalized solutions for parabolic equations (see also [21]), however in our opinion in the case of Orlicz spaces that this result is not known.

Recently, Gwiazda and al. studied the problem 1.1 in the Musielak Orlicz space in [18] but they assumed more restrained conditions on the $N$-function $M$ and $M^{*}$ namely $M(x, \xi) \xi \geq \alpha|\xi|^{1+\eta}$ and $M^{*} \in \Delta_{2}$. In [11] and [17] the authors have studied a problem of the type 1.1 by using the Galerkin classical method to demonstrate the existence of the solution, contrary to our strategy which consists of using the Rothe method to approche the problem 1.1 with a sequences of elliptic problems.

Precisely in this work we use the Rothe's method as a mean ingredient to prove our principal result. Rothe's method introduced by E. Rothe in 1930 and it has been used and developed by many authors, e.g P.P. Mosolov, K. Rektorys in linear and quasilinear 
parabolic problems. Nonlinear and abstract parabolic problems have been studied by Kaũr et al., using similar technique to that of Rothe's method. For the more complete references we refer the reader to $[2,8,9,16-18,20]$ and [32].

Our aim is to prove the existence and uniqueness of an entropy solution for the problem 1.1 in Orlicz spaces using Rothe time-discretization method, which is based on a semidiscretization of the given problem with respect to the time variable.

We will approximate the parabolic problem 1.1 by a sequence of the following elliptic problems:

$$
\left\{\begin{array}{c}
u^{n}-\tau \operatorname{div}\left(a\left(x, \nabla u^{n}\right)\right)+\tau \beta\left(u^{n}\right)=\tau f_{n}+u^{n-1} \text { in } \Omega \\
u^{n}=0 \text { on } \partial \Omega \\
u^{0}=0 \text { in } \Omega .
\end{array}\right.
$$

We will get our results by a semi discretization process, then we prove uniqueness and stability results for the semi-discretized problem. Within the proof we use truncation methods, monotonicity arguments techniques, the integration by parts formula, Young inequality which have been adapted to non-reflexive Orlicz spaces.

This paper is divided into four sections. In the next one, we recall some well-known preliminaries, properties, and results of Orlicz-Sobolev spaces. Section (3) contains the basic assumptions and the main result. Finally, in Sect. (4) we prove the existence and uniqueness of entropy solution to the nonlinear parabolic equation 1.

\section{Preliminaries}

Let $M: \mathbb{R}^{+} \rightarrow \mathbb{R}^{+}$be an $\mathrm{N}$-function, i.e., $M$ is continuous, convex, with $M(t)>0$ for $t>0, \frac{M(t)}{t} \rightarrow 0$ as $t \rightarrow 0$ and $\frac{M(t)}{t} \rightarrow \infty$ as $t \rightarrow \infty$. Equivalently, $M$ admits the representation: $M(t)=\int_{0}^{t} a(\tau) d \tau$ where $a: \mathbb{R}^{+} \rightarrow \mathbb{R}^{+}$is non-decreasing, right continuous, with $a(0)=0, a(t)>0$ for $t>0$ and $a(t) \rightarrow \infty$ as $t \rightarrow \infty$. The N-function $M^{*}$ conjugate to $M$ is defined by $M^{*}(t)=\int_{0}^{t} \bar{a}(\tau) d \tau$, where $\bar{a}: \mathbb{R}^{+} \rightarrow \mathbb{R}^{+}$is given by $\bar{a}(t)=\sup \{s: a(s) \leq t\}$.

The $\mathrm{N}$-function $M$ is said to satisfy the $\Delta_{2}$ condition if, for some $k>0$,

$$
M(2 t) \leq k M(t) \quad \text { for all } t \geq 0 .
$$

When this inequality holds only for $t \geq t_{0}>0, M$ is said to satisfy the $\Delta_{2}$ condition near infinity.

Young's inequality

$$
a . b \leq M(a)+M^{*}(b) \quad \text { for all } a, b \geq 0 .
$$

Let $\Omega$ be an open subset of $\mathbb{R}^{d}$. The Orlicz class $\mathcal{L}_{M}(\Omega)$ (resp. the Orlicz space $L_{M}(\Omega)$ ) is defined as the set of (equivalence classes of) real-valued measurable functions $u$ on $\Omega$ such that: 


$$
\int_{\Omega} M(u(x)) d x<+\infty \quad\left(\operatorname{resp} . \int_{\Omega} M\left(\frac{u(x)}{\lambda}\right) d x<+\infty \text { for some } \lambda>0\right) .
$$

Note that $L_{M}(\Omega)$ is a Banach space under the norm:

$$
\|u\|_{M, \Omega}=\inf \left\{\lambda>0: \int_{\Omega} M\left(\frac{u(x)}{\lambda}\right) d x \leq 1\right\}
$$

and $\mathcal{L}_{M}(\Omega)$ is a convex subset of $L_{M}(\Omega)$. The closure in $L_{M}(\Omega)$ of the set of bounded measurable functions with compact support in $\bar{\Omega}$ is denoted by $E_{M}(\Omega)$. The equality $E_{M}(\Omega)=L_{M}(\Omega)$ holds if and only if $M$ satisfies the $\Delta_{2}$ condition, for all $t$ or for $t$ large according to whether $\Omega$ has infinite measure or not.

The dual of $E_{M}(\Omega)$ can be identified with $L_{M^{*}}(\Omega)$ by means of the pairing $\int_{\Omega} u(x) v(x) d x$, and the dual norm on $L_{M^{*}}(\Omega)$ is equivalent to $\|\cdot\|_{M^{*}, \Omega}$.

The space $L_{M}(\Omega)$ is reflexive if and only if $M$ and $M^{*}$ satisfy the $\Delta_{2}$ condition, for all $t$ or for $t$ large, according to whether $\Omega$ has infinite measure or not.

We now turn to the Orlicz-Sobolev space. $W^{1} L_{M}(\Omega)\left(\operatorname{resp} . W^{1} E_{M}(\Omega)\right)$ is the space of all functions $u$ such that $u$ and its distributional derivatives up to order 1 lie in $L_{M}(\Omega)$ (resp. $E_{M}(\Omega)$ ). This is a Banach space under the norm:

$$
\|u\|_{1, M, \Omega}=\sum_{|\alpha| \leq 1}\left\|D^{\alpha} u\right\|_{M, \Omega}
$$

Thus $W^{1} L_{M}(\Omega)$ and $W^{1} E_{M}(\Omega)$ can be identified with subspaces of the product of $d+1$ copies of $L_{M}(\Omega)$. Denoting this product by $\Pi L_{M}$, we will use the weak topologies $\sigma\left(\Pi L_{M}, \Pi E_{M^{*}}\right)$ and $\sigma\left(\Pi L_{M}, \Pi L_{M^{*}}\right)$. The space $W_{0}^{1} E_{M}(\Omega)$ is defined as the (norm) closure of the Schwartz space $\mathcal{D}(\Omega)$ in $W^{1} E_{M}(\Omega)$ and the space $W_{0}^{1} L_{M}(\Omega)$ as the $\sigma\left(\Pi L_{M}, \Pi E_{M^{*}}\right)$ closure of $\mathcal{D}(\Omega)$ in $W^{1} L_{M}(\Omega)$. We say that $u_{\eta}$ converges to $u$ for the modular convergence in $W^{1} L_{M}(\Omega)$ if for some $\lambda>0, \int_{\Omega} M\left(\frac{D^{\alpha} u_{n}-D^{\alpha} u}{\lambda}\right) d x \rightarrow 0$ for all $|\alpha| \leq 1$. This implies convergence for $\sigma\left(\Pi L_{M}, \Pi L_{M^{*}}\right)$. If $M$ satisfies the $\Delta_{2}$ condition on $\mathbb{R}^{+}$(near infinity only when $\Omega$ has finite measure), then modular convergence coincides with norm convergence.

Let $W^{-1} L_{M^{*}}(\Omega)\left(\right.$ resp. $\left.W^{-1} E_{M^{*}}(\Omega)\right)$ denote the space of distributions on $\Omega$ which can be written as sums of derivatives of order $\leq 1$ of functions in $L_{M^{*}}(\Omega)\left(\operatorname{resp} . E_{M^{*}}(\Omega)\right)$. It is a Banach space under the usual quotient norm.

If the open set $\Omega$ has the segment property, then the space $\mathcal{D}(\Omega)$ is dense in $W_{0}^{1} L_{M}(\Omega)$ for the modular convergence and for the topology $\sigma\left(\Pi L_{M}, \Pi L_{M^{*}}\right)$ (cf.[13]). Consequently, the action of a distribution in $W^{-1} L_{M^{*}}(\Omega)$ on an element of $W_{0}^{1} L_{M}(\Omega)$ is well defined. For more details, see [1, 22].

For $k>0$, we define the truncation at height $k, T_{k}: \mathbb{R} \rightarrow \mathbb{R}$ by

$$
T_{k}(s)=\left\{\begin{array}{lll}
s & \text { if } & |s| \leq k \\
\frac{k s}{|s|} & \text { if } & |s|<k
\end{array}\right.
$$

and its primitive by 


$$
J_{k}(s)=\int_{0}^{s} T_{k}(t) d t=\left\{\begin{array}{lll}
\frac{s^{2}}{2} & \text { if } & |s| \leq k \\
k|s|-\frac{k^{2}}{2} & \text { if } & |s|>k
\end{array}\right.
$$

it is pretty obvious that $J_{k}(s) \geq 0$ and $J_{k}(s) \leq k|s|$.

We have as in the paper [3],

$$
\left\langle\frac{\partial v}{\partial t}, T_{k}(v)\right\rangle_{Q_{T}}=\int_{\Omega} J_{k}(v(t)) d x-\int_{\Omega} J_{k}(v(0)) d x
$$

where $v \in W_{0}^{1, x} L_{M}\left(Q_{T}\right) \cap L^{\infty}\left(Q_{T}\right)$.

Lemma 2.1 [13] Let $F: \mathbb{R} \longrightarrow \mathbb{R}$ be uniformly lipschitzian, with $F(0)=0$. Let $M$ be an $N$-function and let $u \in W^{1} L_{M}(\Omega)\left(\operatorname{resp} . W^{1} E_{M}(\Omega)\right)$. Then $F(u) \in W^{1} L_{M}(\Omega)$ (resp. $W^{1} E_{M}(\Omega)$ ). Moreover, if the set $D$ of discontinuity points of $F^{\prime}$ is finite, then

$$
\frac{\partial}{\partial x_{i}} F(u)=\left\{\begin{array}{c}
F^{\prime}(u) \frac{\partial u}{\partial x_{i}} \quad \text { a.e. in }\{x \in \Omega: u(x) \notin D\} \\
0 \quad \text { a.e. in }\{x \in \Omega: u(x) \in D\}
\end{array}\right.
$$

Lemma 2.2 [13] Let $F: \mathbb{R} \rightarrow \mathbb{R}$ be uniformly lipschitzian, with $F(0)=0$. We suppose that the set of discontinuity points of $F^{\prime}$ is finite. Let $M$ be an $N$-function, then the mapping $F: W^{1} L_{M}(\Omega) \rightarrow W^{1} L_{M}(\Omega)$ is sequentially continuous with respect to the weak * topology $\sigma\left(\Pi L_{M}, \Pi E_{M^{*}}\right)$.

Let $\Omega$ be a bounded open subset of $\mathbb{R}^{d}, T>0$ and set $\left.Q_{T}=\Omega \times\right] 0, T[$. $M$ be an $\mathrm{N}$-function. For each $\alpha \in \mathbb{N}^{d}$, denote by $D_{x}^{\alpha}$ the distributional derivative on $Q$ of order $\alpha$ with respect to the variable $x \in \mathbb{N}^{d}$. The inhomogeneous Orlicz-Sobolev spaces are defined as follows,

$$
W^{1, x} L_{M}\left(Q_{T}\right)=\left\{u \in L_{M}\left(Q_{T}\right): D_{x}^{\alpha} u \in L_{M}\left(Q_{T}\right) \forall|\alpha| \leq 1\right\} .
$$

The last space is Banach spaces under the norm,

$$
\|u\|=\sum_{|\alpha| \leq 1}\left\|D_{x}^{\alpha} u\right\|_{M, Q_{T}} .
$$

We can easily show that they form a complementary system when $\Omega$ satisfies the segment property. These spaces are considered as subspaces of the product space $\Pi L_{M}\left(Q_{T}\right)$ which have as many copies as there is $\alpha$-order derivatives, $|\alpha| \leq 1$. We shall also consider the weak topologies $\sigma\left(\Pi L_{M}, \Pi E_{M^{*}}\right)$ and $\sigma\left(\Pi L_{M}, \Pi L_{M^{*}}\right)$. If $u \in W^{1, x} L_{M}\left(Q_{T}\right)$ then the function $: t \longmapsto u(t)=u(t,$.$) is defined on [0, T]$ with values in $W^{1} L_{M}(\Omega)$. If, further, $u \in W^{1, x} E_{M}\left(Q_{T}\right)$ then the concerned function is a $W^{1} E_{M}(\Omega)$-valued and is strongly measurable. Furthermore the following imbedding holds: $W^{1, x} E_{M}\left(Q_{T}\right) \subset L^{1}\left(0, T ; W^{1} E_{M}(\Omega)\right)$. The space $W^{1, x} L_{M}\left(Q_{T}\right)$ is not in general separable, if $u \in W^{1, x} L_{M}\left(Q_{T}\right)$, we can not conclude that the function $u(t)$ is measurable on $[0, T]$. However, the scalar function $t \mapsto\|u(t)\|_{M, \Omega}$ is in $L^{1}(0, T)$. The space $W_{0}^{1, x} E_{M}\left(Q_{T}\right)$ is defined as the (norm) closure in $W^{1, x} E_{M}\left(Q_{T}\right)$ of $\mathcal{D}\left(Q_{T}\right)$. We can easily 
show as in [14] that when $\Omega$ has the segment property, then each element $u$ of the closure of $\mathcal{D}\left(Q_{T}\right)$ with respect of the weak * topology $\sigma\left(\Pi L_{M}, \Pi E_{M^{*}}\right)$ is a limit, in $W^{1, x} L_{M}\left(Q_{T}\right)$, of some subsequence $\left(u_{i}\right) \subset \mathcal{D}\left(Q_{T}\right)$ for the modular convergence; i.e., there exists $\lambda>0$ such that for all $|\alpha| \leq 1$,

$$
\int_{Q} M\left(\frac{D_{x}^{\alpha} u_{i}-D_{x}^{\alpha} u}{\lambda}\right) d x d t \rightarrow 0 \text { as } i \rightarrow \infty
$$

This implies that $\left(u_{i}\right)$ converges to $u$ in $W^{1, x} L_{M}\left(Q_{T}\right)$ for the weak topology $\sigma\left(\Pi L_{M}, \Pi L_{M^{*}}\right)$. Consequently,

$$
{\overline{\mathcal{D}\left(Q_{T}\right)}}^{\sigma\left(\Pi L_{M}, \Pi E_{M^{*}}\right)}={\overline{\mathcal{D}\left(Q_{T}\right)}}^{\sigma\left(\Pi L_{M}, \Pi L_{M^{*}}\right)} .
$$

This space will be denoted by $W_{0}^{1, x} L_{M}\left(Q_{T}\right)$. Furthermore, $W_{0}^{1, x} E_{M}\left(Q_{T}\right)=W_{0}^{1, x} L_{M}\left(Q_{T}\right) \cap \Pi E_{M}$. Poincaré's inequality also holds in $W_{0}^{1, x} L_{M}\left(Q_{T}\right)$, i.e., there is a constant $C>0$ such that for all $u \in W_{0}^{1, x} L_{M}\left(Q_{T}\right)$ one has,

$$
\sum_{|\alpha| \leq 1}\left\|D_{x}^{\alpha} u\right\|_{M, Q_{T}} \leq C \sum_{|\alpha|=1}\left\|D_{x}^{\alpha} u\right\|_{M, Q_{T}}
$$

Thus both sides of the last inequality are equivalent norms on $W_{0}^{1, x} L_{M}\left(Q_{T}\right)$. We have then the following complementary system

$$
\left(\begin{array}{ll}
W_{0}^{1, x} L_{M}\left(Q_{T}\right) & F \\
W_{0}^{1, x} E_{M}\left(Q_{T}\right) & F_{0}
\end{array}\right)
$$

$F$ being the dual space of $W_{0}^{1, x} E_{M}\left(Q_{T}\right)$. It is also, except for an isomorphism, the quotient of $\Pi L_{M^{*}}$ by the polar set $W_{0}^{1, x} E_{M}\left(Q_{T}\right)^{\perp}$, and will be denoted by $F=W^{-1, x} L_{M^{*}}\left(Q_{T}\right)$ and it is shown that,

$$
W^{-1, x} L_{M^{*}}\left(Q_{T}\right)=\left\{f=\sum_{|\alpha| \leq 1} D_{x}^{\alpha} f_{\alpha}: f_{\alpha} \in L_{M^{*}}\left(Q_{T}\right)\right\} .
$$

This space will be equipped with the usual quotient norm

$$
\|f\|=\inf \sum_{|\alpha| \leq 1}\left\|f_{\alpha}\right\|_{M^{*}, Q}
$$

where the infimum is taken on all possible decompositions

$$
f=\sum_{|\alpha| \leq 1} D_{x}^{\alpha} f_{\alpha}, \quad f_{\alpha} \in L_{M^{*}}\left(Q_{T}\right) .
$$

The space $F_{0}$ is then given by,

$$
F_{0}=\left\{f=\sum_{|\alpha| \leq 1} D_{x}^{\alpha} f_{\alpha}: f_{\alpha} \in E_{M^{*}}\left(Q_{T}\right)\right\}
$$

and is denoted by $F_{0}=W^{-1, x} E_{M^{*}}\left(Q_{T}\right)$. 
Remark 2.3 We can easily check, using lemma 2.1, that each uniformly lipschitzian mapping $F$, with $F(0)=0$, acts in inhomogeneous Orlicz-Sobolev spaces $W^{1, x} L_{M}\left(Q_{T}\right)$ and $W_{0}^{1, x} L_{M}\left(Q_{T}\right)$.

In the sequel we have to use the following results which concern mollification with respect to time and space variable and some trace results.

Thus we define, for all $\mu>0$ and all $(x, t) \in Q_{T}$

$$
u_{\mu}(x, t)=\mu \int_{-\infty}^{t} \tilde{u}(x, s) \exp (\mu(s-t)) d s .
$$

\section{Lemma 2.4 [12]}

1. If $u \in L_{M}\left(Q_{T}\right)$ then $u_{\mu} \rightarrow u$ as $\mu \rightarrow+\infty$ in $L_{M}\left(Q_{T}\right)$ for the modular convergence.

2. If $u \in W^{1, x} L_{M}\left(Q_{T}\right)$ then $u_{\mu} \rightarrow u$ as $\mu \rightarrow+\infty$ in $W^{1, x} L_{M}\left(Q_{T}\right)$ for the modular convergence.

3. If $u \in W^{1, x} L_{M}\left(Q_{T}\right)$ then $\frac{\partial u_{\mu}}{\partial t}=\mu\left(u-u_{\mu}\right)$.

Lemma 2.5 [12] Let Mbean $N$-function. Let $\left(u_{n}\right)$ bea sequence of $W^{1, x} L_{M}\left(Q_{T}\right)$ such that, $u_{n} \rightarrow u$ weakly in $W^{1, x} L_{M}\left(Q_{T}\right)$ for $\sigma\left(\Pi L_{M}, \Pi E_{M^{*}}\right)$ and $\frac{\partial u_{n}}{\partial t}=h_{n}+k_{n}$ in $D^{\prime}\left(Q_{T}\right)$ with $h_{n}$ is bounded in $W^{-1, x} L_{M^{*}}\left(Q_{T}\right)$ and $k_{n}$ is bounded in the space $L^{1}\left(Q_{T}\right)$.

Then, $u_{n} \rightarrow u$ strongly in $L_{\text {Loc }}^{1}\left(Q_{T}\right)$.

If further, $u_{n} \in W_{0}^{1, x} L_{M^{*}}\left(Q_{T}\right)$, then $u_{n} \rightarrow$ u strongly in $L^{1}\left(Q_{T}\right)$.

Lemma 2.6 [11] Let $\Omega$ be a bounded open subset of $\mathbb{R}^{d}$ with the segment property.

Then,

$$
\left\{u \in W_{0}^{1, x} L_{M^{*}}\left(Q_{T}\right): \frac{\partial u}{\partial t} \in W^{-1, x} L_{M^{*}}\left(Q_{T}\right)+L^{1}\left(Q_{T}\right)\right\} \subset C\left([0, T], L^{1}(\Omega)\right) .
$$

\section{Remark 2.7 .}

1. The statement of lemma 2.5 generalizes that of Simon in Orlicz-Sobolev Spaces.

2. While lemma 2.6 generalizes the trace theorem in this general setting.

3. Let us mention that the following trace result, hold true: $D\left(Q_{T}\right)$ is dense in $\left\{u \in W_{0}^{1, x} L_{M^{*}}(Q) \cap L^{2}\left(Q_{T}\right): \frac{\partial u}{\partial t} \in W^{-1, x} L_{M^{*}}\left(Q_{T}\right)+L^{2}\left(Q_{T}\right)\right\}$ for the modular convergence,(see [11]), such trace result generalizes the following classical result, i.e., $\left\{u \in L^{2}\left(0, T, H_{0}^{1}(\Omega)\right): \frac{\partial u}{\partial t} \in L^{2}\left(0, T, H^{-1}(\Omega)\right) \subset C\left([0, T], L^{2}(\Omega)\right\}\right.$.

Let $\Omega$ be a bounded open subset of $\mathbb{R}^{d}$ and let $\mathrm{M}$ be an $\mathrm{N}$-function. Consider a second-order partial differential operator $A: D(A) \subset W_{0}^{1} L_{M}(\Omega) \rightarrow W^{-1} L_{M^{*}}(\Omega)$ in divergence form

$$
A(v)=-\operatorname{div}(a(x, \nabla v))
$$

where $a: \Omega \times \mathbb{R}^{d} \rightarrow \mathbb{R}^{d}$ is a Caratheodory function satisfying for almost every $x \in \Omega$, and for every $v, v^{*} \in D(A)$, such that $\nabla v \neq \nabla v^{*}$, we have

$$
|a(x, \nabla v)| \leq \gamma\left[c(x)+M^{*-1} M(|\nabla v|)\right]
$$




$$
\begin{gathered}
{\left[a(x, \nabla v)-a\left(x, \nabla v^{*}\right)\right]\left[\nabla v-\nabla v^{*}\right]>0} \\
a(x, \nabla v) \nabla v>\alpha M(|\nabla v|),
\end{gathered}
$$

where $c(x) \in E_{M^{*}}(\Omega), \alpha, \gamma>0$ are a real numbers.

We have the following lemma:

Lemma 2.8 [26] Under assumptions (10-12), and let $\left(z_{n}\right)$ be a sequence in $W_{0}^{1, x} L_{M}(\Omega)$ such that,

$$
\begin{gathered}
z_{n} \rightarrow z \text { in } W_{0}^{1, x} L_{M}(\Omega) \text { for } \sigma\left(\Pi L_{M}(\Omega), \Pi E_{M^{*}}(\Omega)\right), \\
\left(a\left(x, \nabla z_{n}\right)\right)_{n} \text { is bounded in }\left(L_{M^{*}}(\Omega)\right)^{d}, \\
\int_{\Omega}\left[a\left(x, \nabla z_{n}\right)-a\left(x, \nabla z \chi_{s}\right)\right]\left[\nabla z_{n}-\nabla z \chi_{s}\right] d x \longrightarrow 0,
\end{gathered}
$$

as $n$ and $s$ tend to $+\infty$, and where $\chi_{s}$ is the characteristic function of

$$
\Omega_{s}=\{x \in \Omega ;|\nabla z| \leq s\}
$$

Then,

$$
\begin{gathered}
\nabla z_{n} \rightarrow \nabla z \text { a.e. in } \Omega . \\
\lim _{n \rightarrow \infty} \int_{\Omega} a\left(x, \nabla z_{n}\right) \nabla z_{n} d x=\int_{\Omega} a(x, \nabla z) \nabla z d x \\
M\left(\left|\nabla z_{n}\right|\right) \rightarrow M(|\nabla z|) \text { in } L^{1}(\Omega) .
\end{gathered}
$$

Consider the following non-linear elliptic problem:

$$
\left\{\begin{array}{c}
-\operatorname{div}(a(x, \nabla u))+g(u)=F \text { in } \Omega \\
u=0 \text { on } \partial \Omega
\end{array}\right.
$$

where

$g$ is a continuous and non-decreasing function on $\mathbb{R}$ such that $g(0)=0$.

$$
F \in L^{1}(\Omega) \text {. }
$$

Proposition 2.9 Assume that (10-12) and (20-21) hold true, then the following problem 


$$
\left\{\begin{array}{c}
T_{k}(u) \in W_{0}^{1} L_{M}(\Omega) \text { for all } k \geq 0, g(u) \in L^{1}(\Omega) \\
\int_{\Omega} a((x, \nabla u)) \nabla T_{k}(u-\varphi) d x+\int_{\Omega} g(u) T_{k}(u-\varphi) d x \leq \int_{\Omega} F T_{k}(u-\varphi) d x \\
\text { for all } \varphi \in W_{0}^{1} L_{M}(\Omega) \cap L^{\infty}(\Omega)
\end{array}\right.
$$

has at least one solution $u$.

Proof Consider the following approximate problems:

$$
\left\{\begin{array}{c}
u_{n} \in W_{0}^{1} L_{M}(\Omega) \\
\int_{\Omega} a\left(\left(x, \nabla u_{n}\right)\right) \nabla v d x+\int_{\Omega} g_{n}\left(u_{n}\right) v d x=\int_{\Omega} F_{n} v d x \\
\text { for all } v \in W_{0}^{1} L_{M}(\Omega)
\end{array}\right.
$$

where $\left(F_{n}\right)_{n} \subset W^{-1} E_{M}(\Omega)$ is a sequence of smooth functions such that $F_{n} \longrightarrow F$ in $L^{1}(\Omega)$ and $g_{n}(s)=T_{n}(g(s))$.

Note that $g_{n}(s) s \geq 0,\left|g_{n}(s)\right| \leq n$ and $\left|g_{n}(s)\right| \leq|g(s)|$.

Since $g_{n}$ is bounded for any fixed $n>0$, there exists at least one solution $u_{n}$ of the problem (2.21) (see Proposition 13 in [15]).

Using the same technics and the same steps as in the paper [10], we deduce that the problem (2.17) has at least one solution.

\section{Assumptions and main result}

\subsection{Assumptions}

Let $\Omega$ be a bounded open set of $\mathbb{R}^{d}(d \geq 2)$ with the segment property, $T>0$ is given real number and $\left.Q_{T}=\right] 0 . T[\times \Omega$.

Consider a second order partial differential operator $A: D(A) \subset W^{1, x} L_{M}\left(Q_{T}\right) \rightarrow W^{-1, x} L_{M^{*}}\left(Q_{T}\right)$ in divergence $A(u)=-\operatorname{div}(a(x, \nabla u(x, t)))$, where $\mathrm{M}$ is an $\mathrm{N}$-function without assuming a $\Delta_{2}$-condition on $\mathrm{M}$.

We consider the following nonlinear parabolic problem:

$$
\left\{\begin{array}{c}
\left.\frac{\partial u}{\partial t}-\operatorname{div}(a(x, \nabla u(x, t)))+\beta(u)=f \text { in } Q_{T}=\right] 0 . T[\times \Omega \\
\left.u=0 \text { on } \Sigma_{T}=\right] 0 . T[\times \partial \Omega \\
u(0, .)=0 \text { in } \Omega
\end{array}\right.
$$

where $a: \Omega \times \mathbb{R}^{d} \rightarrow \mathbb{R}^{d}$ Caratheodory function satisfying for almost every $(x, t) \in Q_{T}$, and for every $v, v^{*} \in D(A)$, such that $\nabla v(x, t) \neq \nabla v^{*}(x, t)$ in $\mathbb{R}^{d}$,

$$
|a(x, \nabla v(x, t))| \leq \gamma\left[c(x, t)+M^{*-1} M(|\nabla v(x, t)|)\right]
$$




$$
\begin{gathered}
{\left[a(x, \nabla v(x, t))-a\left(x, \nabla v^{*}(x, t)\right)\right]\left[\nabla v(x, t)-\nabla v^{*}(x, t)\right]>0} \\
a(x, \nabla v(x, t)) \nabla v(x, t)>\alpha M(|\nabla v(x, t)|),
\end{gathered}
$$

where $c(x, t) \in E_{M^{*}}\left(Q_{T}\right), \alpha, \gamma>0$ are a real numbers.

$$
\text { The data } f \in L^{1}\left(Q_{T}\right) \text {, }
$$

$\beta$ is non decreasing continuous functions on $\mathbb{R}$ such that $\beta(0)=0$.

Remark 3.1 We can take $u(0,)=.u_{0}$ such that $u_{0} \in L^{1}(\Omega)$ and we use regularization as in [25]

Throughout this paper $\langle.,$.$\rangle means for either the pairing between$ $W_{0}^{1} L_{M}\left(Q_{T}\right) \cap L^{\infty}\left(Q_{T}\right)$ and $W^{-1} L_{M^{*}}\left(Q_{T}\right)+L^{1}(Q)$ or between $W_{0}^{1} L_{M}\left(Q_{T}\right)$ and $W^{-1} L_{M^{*}}\left(Q_{T}\right)$. And also for simplicity, we write $a(x, \nabla u)$ instead of $a(x, \nabla u(x, t))$.

Lemma 3.2 Under assumptions (24-26), and let $\left(z_{n}\right)$ be a sequence in $W_{0}^{1, x} L_{M}\left(Q_{T}\right)$ such that,

$$
\begin{gathered}
z_{n} \rightarrow z \text { in } W_{0}^{1, x} L_{M}\left(Q_{T}\right) \text { for } \sigma\left(\Pi L_{M}\left(Q_{T}\right), \Pi E_{M^{*}}\left(Q_{T}\right)\right), \\
\left(a\left(x, t, \nabla z_{n}\right)\right)_{n} \text { is bounded in }\left(L_{M^{*}}\left(Q_{T}\right)\right)^{d}, \\
\int_{Q_{T}}\left[a\left(x, t, \nabla z_{n}\right)-a\left(x, t, \nabla z \chi_{s}\right)\right]\left[\nabla z_{n}-\nabla z \chi_{s}\right] d x d t \longrightarrow 0,
\end{gathered}
$$

as $n$ and $s$ tend to $+\infty$, and where $\chi_{s}$ is the characteristic function of

$$
Q_{s}=\left\{(x, t) \in Q_{T} ;|\nabla z| \leq s\right\}
$$

Then,

$$
\begin{gathered}
\nabla z_{n} \rightarrow \nabla z \text { a.e. in } Q_{T} . \\
\lim _{n \rightarrow \infty} \int_{Q_{T}} a\left(x, t, \nabla z_{n}\right) \nabla z_{n} d x d t=\int_{Q_{T}} a(x, t, \nabla z) \nabla z d x d t, \\
M\left(\left|\nabla z_{n}\right|\right) \rightarrow M(|\nabla z|) \text { in } L^{1}\left(Q_{T}\right),
\end{gathered}
$$

Proof Using the same argument in [3] we get the result. 


\subsection{The semi-discrete problem}

By using the semi-discretization in time by the implicit Euler method for the parabolic problem (1) we get,

$$
\left\{\begin{array}{c}
u^{n}-\tau \operatorname{div}\left(a\left(x, \nabla u^{n}\right)\right)+\tau \beta\left(u^{n}\right)=\tau f_{n}+u^{n-1} \text { in } \Omega \\
u^{n}=0 \text { on } \partial \Omega
\end{array}\right.
$$

where $N \tau=T, 1 \leq n \leq N$ and $f_{n}()=.\frac{1}{\tau} \int_{(n-1) \tau}^{n \tau} f(s,) d$.$s .$

We will sometimes use the shorter notation $\|.\|_{1}$ instead of $\|\cdot\|_{L^{1}(\Omega)}$.

Definition 3.3 An entropy solution to the discretized problems (35) is a sequence of measurable functions $\left(u^{n}\right)_{0 \leq n \leq N}$ such that $u^{0}=0$ and $u^{n}$ is defined by induction as an entropy solution of the problem

$$
\left\{\begin{array}{c}
u^{n}-\tau \operatorname{div}\left(a\left(x, \nabla u^{n}\right)\right)+\tau \beta\left(u^{n}\right)=\tau f_{n}+u^{n-1} \text { in } \Omega \\
u^{n}=0 \text { on } \partial \Omega .
\end{array}\right.
$$

i.e for all $1 \leq n \leq N$ and $k>0$ we have $T_{k}\left(u^{n}\right) \in W_{0}^{1} L_{M}(\Omega)$ and for all $\varphi \in W_{0}^{1} L_{M}(\Omega) \cap L^{\infty}(\Omega)$ we have

$$
\begin{aligned}
& \int_{\Omega} u^{n} T_{k}\left(u^{n}-\varphi\right) d x+\tau \int_{\Omega} a\left(x, \nabla u^{n}\right) \nabla T_{k}\left(u^{n}-\varphi\right) d x+\int_{\Omega} \tau \beta\left(u^{n}\right) T_{k}\left(u^{n}-\varphi\right) d x \\
& \quad \leq \int_{\Omega}\left(\tau f_{n}+u^{n-1}\right) T_{k}\left(u^{n}-\varphi\right) d x .
\end{aligned}
$$

Lemma 3.4 Let $\left(u^{n}\right)_{0 \leq n \leq N}, N \in \mathbb{N}$ be an entropy solution of the approximate problem (35), then for all $n=1, \ldots, N$, we have $u^{n} \in L^{1}(\Omega)$.

Proof Let us take $\varphi=0$ as test function in (37) in case of $n=1$, we get

$$
\int_{\Omega}\left(\tau \beta\left(u^{1}\right)+u^{1} T_{k}\left(u^{1}\right) d x+\tau \int_{\Omega} a\left(x, \nabla u^{1}\right) \nabla T_{k}\left(u^{1}\right) d x \leq \int_{\Omega}\left(\tau f_{1}+u^{0}\right) T_{k}\left(u^{1}\right) d x\right.
$$

from the definition (3.3), we have $u^{0}=0$, this implies

$$
\begin{aligned}
& \int_{\Omega}\left(\tau \beta\left(u^{1}\right) T_{k}\left(u^{1}\right) d x+\int_{\Omega} u^{1} T_{k}\left(u^{1}\right) d x+\tau \int_{\Omega} a\left(x, \nabla u^{1}\right) \nabla T_{k}\left(u^{1}\right) d x\right. \\
& \quad \leq \int_{\Omega} \tau f_{1} T_{k}\left(u^{1}\right) d x .
\end{aligned}
$$


By assumption (28) and the properties of $T_{k}$, we obtain

$$
\int_{\Omega} \tau \beta\left(u^{1}\right) T_{k}\left(u^{1}\right) d x \geq 0 .
$$

From the assumption (26), we obtain

$$
\int_{\Omega} a\left(x, \nabla u^{1}\right) \nabla T_{k}\left(u^{1}\right) d x \geq 0
$$

On the other hand, for all $1 \leq n \leq N$, we have $\tau f_{n}()=.\int_{(n-1) \tau}^{n \tau} f(t,) d$.$t in \Omega$, this implies that

$$
\begin{aligned}
\int_{\Omega} \tau f_{1}(x) T_{k}\left(u^{1}\right) d x & =\int_{\Omega} \int_{0}^{\tau} f(t, x) T_{k}\left(u^{1}\right) d t d x \\
& \leq \int_{\Omega} \int_{0}^{T}|f(t, x)|\left|T_{k}\left(u^{1}\right)\right| d t d x \\
& \leq k \int_{Q_{T}}|f(t, x)| d t d x \\
& \leq k|| f \|_{L^{1}\left(Q_{T}\right)} .
\end{aligned}
$$

Thus, we remark that

$$
u^{1} T_{k}\left(u^{1}\right) \geq 0 .
$$

By combining the results (39-40) and (43), we obtain

$$
0 \leq \int_{\Omega} u^{1} T_{k}\left(u^{1}\right) d x \leq k|| f \|_{L^{1}\left(Q_{T}\right)},
$$

this implies that

$$
0 \leq \int_{\Omega} u^{1} \frac{T_{k}\left(u^{1}\right)}{k} d x \leq\|f\|_{L^{1}\left(Q_{T}\right)} .
$$

Since $u^{1} \frac{T_{k}\left(u^{1}\right)}{k} \geq 0$, and by using the Fatou's lemma with $k \rightarrow 0$, we deduce that

$$
\left\|u^{1}\right\|_{1} \leq C
$$

with $C$ not depending on $k$. 
Hence, by induction, in the same techniques used in $n=1$, we obtain

$$
\begin{aligned}
& \int_{\Omega}\left(\tau \beta\left(u^{n}\right) T_{k}\left(u^{n}\right) d x+\int_{\Omega} u^{n} T_{k}\left(u^{n}\right) d x+\tau \int_{\Omega} a\left(x, \nabla u^{n}\right) \nabla T_{k}\left(u^{n}\right) d x\right. \\
& \quad \leq \int_{\Omega} \tau f_{n} T_{k}\left(u^{n}\right) d x+\int_{\Omega} u^{n-1} T_{k}\left(u^{n}\right) d x,
\end{aligned}
$$

We suppose that $u^{n-1} \in L^{1}(\Omega)$, then $\int_{\Omega} u^{n-1} T_{k}\left(u^{n}\right) d x \leq k C$.

Using the same manner as the case $n=1$, we deduce that $u^{n} \in L^{1}(\Omega)$.

Lemma 3.5 Let $\left(u^{n}\right)_{0 \leq n \leq N}, N \in \mathbb{N}$ be an entropy solution of the approximate problem (2), then for all $k>0, n=1 . . N$ and $h>0$, we have

$$
\tau \int_{\left\{h \leq\left|u^{n}\right| \leq h+k\right\}} M\left(\left|\nabla u^{n}\right|\right) d x \leq k \int_{\left\{\left|u^{n}\right| \geq h\right\}} \tau\left|f_{n}\right| d x+k \int_{\left\{\left|u^{n}\right| \geq h\right\}}\left|u^{n-1}\right| d x .
$$

Proof We take $\varphi=T_{h}\left(u^{n}\right)$ as test function in (37), we obtain

$$
\begin{aligned}
& \int_{\Omega} u^{n} T_{k}\left(u^{n}-T_{h}\left(u^{n}\right)\right) d x+\tau \int_{\Omega} a\left(x, \nabla u^{n}\right) \nabla T_{k}\left(u^{n}-T_{h}\left(u^{n}\right)\right) d x \\
& \quad+\int_{\Omega} \tau \beta\left(u^{n}\right) T_{k}\left(u^{n}-T_{h}\left(u^{n}\right)\right) d x \\
& \quad \leq \int_{\Omega}\left(\tau f_{n}+u^{n-1}\right) T_{k}\left(u^{n}-T_{h}\left(u^{n}\right)\right) d x .
\end{aligned}
$$

Since $T_{h}\left(u^{n}\right)=u^{n}$ in $\left\{x \in \Omega,\left|u^{n}\right| \leq h\right\}$, we have

$$
\begin{aligned}
& \int_{\left\{\left|u^{n}\right| \geq h\right\}} u^{n} T_{k}\left(u^{n}-h \cdot \operatorname{sgn}\left(u^{n}\right)\right) d x+\tau \int_{\left\{\left|u^{n}\right| \geq h\right\}} a\left(x, \nabla u^{n}\right) \nabla T_{k}\left(u^{n}-h \cdot \operatorname{sgn}\left(u^{n}\right)\right) d x \\
& \quad+\int_{\left\{\left|u^{n}\right| \geq h\right\}} \tau \beta\left(u^{n}\right) T_{k}\left(u^{n}-h \cdot \operatorname{sgn}\left(u^{n}\right)\right) d x \\
& \quad \leq \int_{\left\{\left|u^{n}\right| \geq h\right\}}\left(\tau f_{n}+u^{n-1}\right) T_{k}\left(u^{n}-h \cdot \operatorname{sgn}\left(u^{n}\right)\right) d x,
\end{aligned}
$$

where $\operatorname{sgn}(s)=\frac{s}{|s|}$.

For $\left|u^{n}\right| \geq h$ we have $\operatorname{sgn}\left(u^{n}\right)=\operatorname{sgn}\left(u^{n}-h \cdot \operatorname{sgn}\left(u^{n}\right)\right)=\operatorname{sgn}\left(T_{k}\left(u^{n}-h \cdot \operatorname{sgn}\left(u^{n}\right)\right)\right)$, this implies that

$$
u^{n} T_{k}\left(u^{n}-h \cdot \operatorname{sgn}\left(u^{n}\right)\right) \geq 0,
$$

then 


$$
\int_{\Omega} u^{n} T_{k}\left(u^{n}-T_{h}\left(u^{n}\right)\right) d x \geq 0 .
$$

In the same way and using hypothesis (28), we obtain

$$
\int_{\Omega} \tau \beta\left(u^{n}\right) T_{k}\left(u^{n}-T_{h}\left(u^{n}\right)\right) d x \geq 0 .
$$

Moreover, we have

$$
\int_{\Omega} a\left(x, \nabla u^{n}\right) \nabla T_{k}\left(u^{n}-T_{h}\left(u^{n}\right)\right) d x=\int_{\left\{h \leq\left|u^{n}\right| \leq h+k\right\}} a\left(x, \nabla u^{n}\right) \nabla u^{n} d x .
$$

From (50-53), we get

$$
\tau \int_{\left\{h \leq\left|u^{n}\right| \leq h+k\right\}} a\left(x, \nabla u^{n}\right) \nabla u^{n} d x \leq k \int_{\left\{\left|u^{n}\right| \geq h\right\}} \tau\left|f_{n}\right| d x+k \int_{\left\{\left|u^{n}\right| \geq h\right\}}\left|u^{n-1}\right| d x .
$$

Proposition 3.6 Under assumptions (24)-(28), for all $N \in \mathbb{N}$, the approximate problem (35) has a unique entropy solution $u^{n}$.

Proof Let $n=1, \ldots, N$, we tike $F=\tau f_{n}+u^{n-1}$ and $g(s)=\tau \beta(s)+s$, these functions satisfies the conditions of the proposition (2.9), then the problem (25) has an entropy solution $\left(u^{n}\right)_{0 \leq n \leq N}$.

Now let's show the uniqueness: let $\left(u^{n}\right)$ and $\left(v^{n}\right)$ be two entropy solutions of problems (35). For $n=1$, we have

$$
\begin{aligned}
& \int_{\Omega} u^{1} T_{k}\left(u^{1}-\varphi\right) d x+\tau \int_{\Omega} a\left(x, \nabla u^{1}\right) \nabla T_{k}\left(u^{1}-\varphi\right) d x+\int_{\Omega} \tau \beta\left(u^{1}\right) T_{k}\left(u^{1}-\varphi\right) d x \\
& \quad \leq \int_{\Omega}\left(\tau f_{1}+u^{0}\right) T_{k}\left(u^{1}-\varphi\right) d x
\end{aligned}
$$

and

$$
\begin{aligned}
& \int_{\Omega} v^{1} T_{k}\left(v^{1}-\varphi\right) d x+\tau \int_{\Omega} a\left(x, \nabla v^{1}\right) \nabla T_{k}\left(v^{1}-\varphi\right) d x+\int_{\Omega} \tau \beta\left(v^{1}\right) T_{k}\left(v^{1}-\varphi\right) d x \\
& \quad \leq \int_{\Omega}\left(\tau f_{1}+v^{0}\right) T_{k}\left(v^{1}-\varphi\right) d x
\end{aligned}
$$


with $v^{0}=u^{0}=0$.

For simplicity, we write $u=u^{1}$ and $v=v^{1}$.

Let $h>k$, for the solution $u$ we take $\varphi=T_{h}(v)$ as test function, and for the solution $v$ we take $\varphi=T_{h}(u)$ as test function.

By summing up the two inequalities, and letting $h$ go to infinity, we find by applying Lebesque's theorem that

$$
\int_{\Omega}(u-v) T_{k}(u-v) d x+\tau \lim _{h \rightarrow \infty} I_{k, h}+\tau \lim _{h \rightarrow \infty} J_{k, h} \leq 0,
$$

where

$$
I_{k, h}=\int_{\Omega} a(x, \nabla u) \nabla T_{k}\left(u-T_{h}(v)\right) d x+\int_{\Omega} a(x, \nabla v) \nabla T_{k}\left(v-T_{h}(u)\right) d x,
$$

and

$$
J_{k, h}=\int_{\Omega} \beta(u) T_{k}\left(u-T_{h}(v)\right) d x+\int_{\Omega} \beta(v) T_{k}\left(v-T_{h}(u)\right) d x .
$$

By applying hypothesis (28), we get

$$
\lim _{h \rightarrow \infty} J_{k, h}=\int_{\Omega}(\beta(u)-\beta(v)) T_{k}(u-v) d x \geq 0 .
$$

Now, we show that $\lim _{h \rightarrow \infty} I_{k, h} \geq 0$. We take

$$
\begin{gathered}
\Omega_{1}=\{|u|<h,|v|<h\} ; \Omega_{2}=\{|u|<h,|v| \geq h\} \\
\Omega_{3}=\{|u| \geq h,|v|<h\} ; \Omega_{4}=\{|u| \geq h,|v| \geq h\},
\end{gathered}
$$

and we spilt

$$
I_{k, h}=I_{k, h}^{1}+I_{k, h}^{2}+I_{k, h}^{3}+I_{k, h}^{4},
$$

where

$$
I_{k, h}^{i}=\int_{\Omega_{i}} a(x, \nabla u) \nabla T_{k}\left(u-T_{h}(v)\right) d x+\int_{\Omega_{i}} a(x, \nabla v) \nabla T_{k}\left(v-T_{h}(u)\right) d x,(i=1,2,3,4) .
$$

- We show that $I_{k, h}^{1} \geq 0$ : We have 


$$
\begin{aligned}
I_{k, h}^{1} & =\int_{\Omega_{1}}\left[a(x, \nabla u) \nabla T_{k}\left(u-T_{h}(v)\right)+a(x, \nabla v) \nabla T_{k}\left(v-T_{h}(u)\right)\right] d x \\
& =\int_{\Omega_{1}}\left[a(x, \nabla u) \nabla T_{k}(u-v)+a(x, \nabla v) \nabla T_{k}(v-u)\right] d x \\
& =\int_{\Omega_{1} \cap\{|u-v| \leq k\}}(a(x, \nabla u)-a(x, \nabla v))(\nabla u-\nabla v) d x,
\end{aligned}
$$

by using (3.2), we get

$$
I_{k, h}^{1} \geq 0
$$

- We show that $\lim _{h \rightarrow \infty} I_{k, h}^{2} \geq 0$ : We have

$$
I_{k, h}^{2}=\int_{\Omega_{2}}\left[a(x, \nabla u) \nabla T_{k}\left(u-T_{h}(v)\right)+a(x, \nabla v) \nabla T_{k}\left(v-T_{h}(u)\right)\right] d x,
$$

from (26), we have

$$
\int_{\Omega_{2}} a(x, \nabla u) \nabla T_{k}\left(u-T_{h}(v)\right) d x=\int_{\Omega_{2} \cap\left\{\left|u-T_{h}(v)\right| \leq k\right\}} a(x, \nabla u) \nabla u d x \geq 0 .
$$

On the other hand

$$
\begin{aligned}
\int_{\Omega_{2}} a(x, \nabla v) \nabla T_{k}\left(v-T_{h}(u)\right) d x & =\int_{\Omega_{2} \cap\{|u-v| \leq k\}} a(x, \nabla v)(\nabla v-\nabla u) d x \\
& =\int_{\Omega_{2} \cap\{|u-v| \leq k\}} a(x, \nabla v) \nabla v d x-\int_{\Omega_{2} \cap\{|u-v| \leq k\}} a(x, \nabla v) \nabla u d x .
\end{aligned}
$$

Let $0<\theta<\min \left(\frac{1}{2}, \frac{1}{2 \gamma}\right)$, we have

$$
\begin{aligned}
& \frac{1}{\theta} \int_{\Omega_{2} \cap\{|u-v| \leq k\}} \theta a(x, \nabla v) \nabla u d x \leq \frac{1}{\theta} \int_{\Omega_{2} \cap\{|u-v| \leq k\}} M^{*}(|\theta a(x, \nabla v)|) d x \\
& +\frac{1}{\theta} \int_{\Omega_{2} \cap\{|u-v| \leq k\}} M(|\nabla u|) d x,
\end{aligned}
$$

then from (24) and the convexity of $M^{*}$,

$$
\begin{aligned}
& \frac{1}{\theta} \int_{\Omega_{2} \cap\{|u-v| \leq k\}} M^{*}(|\theta a(x, \nabla v)|) d x \\
& \quad \leq \frac{1}{\theta} \int_{\Omega_{2} \cap\{|u-v| \leq k\}} M^{*}(\theta \gamma c(x))+\theta M^{*-1} M(|\nabla v|) d x \\
& \quad \leq \frac{1}{\theta} \int_{\Omega_{2} \cap\{|u-v| \leq k\}} \frac{1}{2} M^{*}(2 \theta \gamma c(x))+\frac{1}{2} M^{*}\left(2 \theta M^{*-1} M(|\nabla v|) d x\right. \\
& \quad \leq \gamma \int_{\Omega_{2} \cap\{|u-v| \leq k\}} M^{*}(c(x))+\int_{\Omega_{2} \cap\{|u-v| \leq k\}} M(|\nabla v|) d x,
\end{aligned}
$$


we deduce that

$$
\begin{aligned}
\left|\int_{\Omega_{2}} a(x, \nabla v) \nabla u d x\right| \leq \gamma & \int_{\Omega_{2} \cap\{|u-v| \leq k\}} M^{*}(c(x)) d x+\int_{\Omega_{2.1}} M(|\nabla v|) d x \\
& +\frac{1}{\theta} \int_{\Omega_{2.2}} M(|\nabla u|) d x,
\end{aligned}
$$

where $\Omega_{2.1}=\{h \leq|v| \leq h+k\}$ and $\Omega_{2.2}=\{h-k \leq|u| \leq h\}$.

According to (48), we have

$$
\tau \int_{\Omega_{2.1}} M(|\nabla v|) d x \leq k \int_{\{|v|>h\}} \tau\left|f_{n}\right| d x+k \int_{\{|v|>h\}}\left|v^{0}\right| d x,
$$

and

$$
\tau \int_{\Omega_{2.2}} M(|\nabla u|) d x \leq k \int_{\{|u|>h-k\}} \tau\left|f_{n}\right| d x+k \int_{\{|u|>h-k\}}\left|u^{0}\right| d x,
$$

since $f_{n} \in L^{1}(\Omega), \quad u^{n} \in L^{1}(\Omega)$ for all $n \in \mathbb{N}, u^{0}=v^{0}=0$ and the fact that $\lim _{h \rightarrow \infty}|\{|v|>h\}|=0, \lim _{h \rightarrow \infty}|\{|u|>h-k\}|=0$ and $c(x) \in E_{M^{*}}$, then

$$
\begin{aligned}
& \lim _{h \rightarrow \infty} \int_{\Omega_{2.1}} M(|\nabla v|) d x=0, \lim _{h \rightarrow \infty} \int_{\Omega_{2.2}} M(|\nabla u|) d x=0 \\
& \text { and } \lim _{h \rightarrow \infty} \int_{\Omega_{2} \cap\{|u-v| \leq k\}} M^{*}(c(x)) d x=0,
\end{aligned}
$$

this implies that

$$
\lim _{h \rightarrow \infty} \int_{\Omega_{2}} a(x, \nabla v) \nabla u d x=0
$$

Hence $\lim _{h \rightarrow \infty} I_{k, h}^{2} \geq 0$.

- We show that $\lim _{h \rightarrow \infty} I_{k, h}^{3} \geq 0$ : We have

$$
I_{k, h}^{3}=\int_{\Omega_{3}} a(x, \nabla u) \nabla T_{k}\left(u-T_{h}(v)\right)+a(x, \nabla v) \nabla T_{k}\left(v-T_{h}(u)\right) d x,
$$

and as

$$
\int_{\Omega_{3}} a(x, \nabla v) \nabla T_{k}\left(v-T_{h}(u)\right) d x=\int_{\Omega_{3} \cap\left\{\left|v-T_{h}(u)\right| \leq k\right\}} a(x, \nabla v) \nabla v d x \geq 0
$$


and

$$
\int_{\Omega_{3}} a(x, \nabla u) \nabla T_{k}\left(u-T_{h}(v)\right) d x=\int_{\Omega_{3} \cap\{|u-v| \leq k\}} a(x, \nabla u)(\nabla u-\nabla v) d x
$$

by same way as used for (71), we deduce that

$$
\lim _{h \rightarrow \infty} \int_{\Omega_{3.1}} M(|\nabla v|) d x=0 \text { and } \lim _{h \rightarrow \infty} \int_{\Omega_{3.1}} M(|\nabla u|) d x=0,
$$

where $\quad \Omega_{3.1}=\{h \leq|v| \leq h+k\} \quad$ and $\quad \Omega_{3.2}=\{h-k \leq|u| \leq h\}$. Hence $\lim _{h \rightarrow \infty} I_{k, h}^{3} \geq 0$.

- We show that $I_{k, h}^{4} \geq 0:$ We have

$$
\begin{aligned}
I_{k, h}^{4} & =\int_{\Omega_{4}} a(x, \nabla u) \nabla T_{k}\left(u-T_{h}(v)\right)+a(x, \nabla v) \nabla T_{k}\left(v-T_{h}(u)\right) d x \\
& =\int_{\Omega_{2} \cap\left\{\left|u-T_{h}(v)\right| \leq k\right\}} a(x, \nabla u) \nabla u d x+\int_{\Omega_{4} \cap\left\{\left|v-T_{h}(u)\right| \leq k\right\}} a(x, \nabla v) \nabla v d x \geq 0 .
\end{aligned}
$$

Hence $I_{k, h} \geq 0$, and as

$$
\int_{\Omega}(u-v) T_{k}(u-v) d x+\tau \lim _{h \rightarrow 0} I_{k, h} \leq 0,
$$

then

$$
\int_{\Omega}(u-v) T_{k}(u-v) d x \leq 0 .
$$

Since

$$
\lim _{k \rightarrow 0} \frac{1}{k} T_{k}(u-v)=\operatorname{sgn}(u-v),
$$

by applying dominated convergence theorem, we get

$$
\|u-v\|_{1} \leq 0 \text {. }
$$

We deduce in the same manner that for $n=1, \ldots, N$,

$$
\left\|u^{n}-v^{n}\right\|_{1} \leq 0
$$

Hence the uniqueness of the solution of the approximate problem (35). 


\subsection{Stability results}

Proposition 3.7 Let $\left(u^{n}\right)_{0 \leq n \leq N}, N \in \mathbb{N}$ be an entropy solution of the approximate problem (35), then there exists a positive constants $C_{1}(f), C_{2}(f)$ and $C(f, k)$ independents on $N$, such that for all $n=1, \ldots N$, we have

(i) $\left\|u^{n}\right\|_{1} \leq C_{1}(f)$

(ii) $\tau \sum_{i=1}^{n}\left\|\beta\left(u^{i}\right)\right\|_{1} \leq C_{1}(f)$

$$
\text { (iii) } \sum_{i=1}^{n}\left\|u^{i}-u^{i-1}\right\|_{1} \leq C_{2}(f)
$$

$$
\text { (iv) } \sum_{i=1}^{n} \tau \int_{\Omega} M\left(\left|\nabla T_{k}\left(u^{i}\right)\right|\right) d x \leq C(f, k),
$$

where $\quad C_{1}=\|f\|_{L^{1}\left(Q_{T}\right)}, \quad C_{2}=\tau \sum_{i=1}^{n}\left(\left\|f_{i}\right\|_{1}+\tau \sum_{i=1}^{n}\left\|\beta\left(u^{i}\right)\right\|_{1}\right.$ and $C(f, k)=\tau k \sum_{i=1}^{n}$ $\left\|f_{i}\right\|_{1}+k \sum_{i=1}^{n}\left\|u^{i}-u^{i-1}\right\|_{1}+\tau k \sum_{i=1}^{n}\left\|\beta\left(u^{i}\right)\right\|_{1}$.

Proof : Proof of (i) and (ii): We take $\varphi=0$ as test function in (37), we obtain

$$
\begin{aligned}
& \int_{\Omega} u^{i} T_{k}\left(u^{i}\right) d x+\tau \int_{\Omega} a\left(x, \nabla u^{i}\right) \nabla T_{k}\left(u^{i}\right) d x+\int_{\Omega} \tau \beta\left(u^{i}\right) T_{k}\left(u^{i}\right) d x \\
& \quad \leq \int_{\Omega}\left(\tau f_{i}\right) T_{k}\left(u^{i}\right) d x+\int_{\Omega}\left(u^{i-1}\right) T_{k}\left(u^{i}\right) d x .
\end{aligned}
$$

Since

$$
\int_{\Omega} a\left(x, \nabla u^{i}\right) \nabla T_{k}\left(u^{i}\right) d x \geq 0
$$

then

$$
\int_{\Omega}\left(\tau \beta\left(u^{i}\right)+u^{i}\right) T_{k}\left(u^{i}\right) d x \leq k \tau \int_{\Omega}\left|f_{i}\right| d x+k \int_{\Omega}\left|u^{i-1}\right| d x,
$$

thus implies that

$$
\int_{\Omega} \frac{u^{i} T_{k}\left(u^{i}\right)}{k} d x+\tau \int_{\Omega} \frac{\beta\left(u^{i}\right) T_{k}\left(u^{i}\right)}{k} d x \leq \tau \int_{\Omega}\left|f_{i}\right| d x+\int_{\Omega}\left|u^{i-1}\right| d x .
$$


Let $k \rightarrow 0$, we get by the properties of $T_{k}$ and the Fatou lemma, that

$$
\tau\left\|\beta\left(u^{i}\right)\right\|_{1}+\left\|u^{i}\right\|_{1} \leq \tau\left\|f_{i}\right\|_{1}+\left\|u^{i-1}\right\|_{1} .
$$

Summing (86) from $i=1$ to $i=n$, we obtain

$$
\tau \sum_{i=1}^{n}\left\|\beta\left(u^{i}\right)\right\|_{1}+\left\|u^{n}\right\|_{1} \leq \sum_{i=1}^{n} \tau\left\|f_{i}\right\|_{1} \leq\|f\|_{L^{1}\left(Q_{T}\right)} .
$$

Proof of (iii): Taking $\varphi=T_{h}\left(u^{i}\right)-\operatorname{sgn}\left(T_{h}\left(u^{i}\right)-T_{h}\left(u^{i-1}\right)\right)$ as test function in (37), we obtain

$$
\begin{aligned}
& \int_{\Omega}\left(u^{i}-u^{i-1}\right) T_{k}\left(u^{i}-T_{h}\left(u^{i}\right)+\operatorname{sgn}\left(T_{h}\left(u^{i}\right)-T_{h}\left(u^{i-1}\right)\right)\right) d x \\
& \quad+\tau \int_{\Omega} a\left(x, \nabla u^{i}\right) \nabla T_{k}\left(u^{i}-T_{h}\left(u^{i}\right)+\operatorname{sgn}\left(T_{h}\left(u^{i}\right)-T_{h}\left(u^{i-1}\right)\right)\right) d x \\
& \quad+\tau \int_{\Omega} \beta\left(u^{i}\right) T_{k}\left(u^{i}-T_{h}\left(u^{i}\right)+\operatorname{sgn}\left(T_{h}\left(u^{i}\right)-T_{h}\left(u^{i-1}\right)\right)\right) d x \\
& \quad \leq \int_{\Omega}\left(\tau f_{i}\right) T_{k}\left(u^{i}-T_{h}\left(u^{i}\right)+\operatorname{sgn}\left(T_{h}\left(u^{i}\right)-T_{h}\left(u^{i-1}\right)\right)\right) d x .
\end{aligned}
$$

We have

$$
\begin{gathered}
\int_{\Omega_{k}^{2}} a\left(x, \nabla u^{i}\right) \nabla T_{k}\left(u^{i}-T_{h}\left(u^{i}\right)+\operatorname{sgn}\left(T_{h}\left(u^{i}\right)-T_{h}\left(u^{i-1}\right)\right)\right) d x=0, \\
\int_{\Omega_{k}^{1} \cap \Omega_{h}^{1}} a\left(x, \nabla u^{i}\right) \nabla T_{k}\left(u^{i}-T_{h}\left(u^{i}\right)+\operatorname{sgn}\left(T_{h}\left(u^{i}\right)-T_{h}\left(u^{i-1}\right)\right)\right) d x=0
\end{gathered}
$$

and

$$
\begin{aligned}
& \int_{\Omega_{k}^{1} \cap \Omega_{h}^{2}} a\left(x, \nabla u^{i}\right) \nabla T_{k}\left(u^{i}-T_{h}\left(u^{i}\right)+\operatorname{sgn}\left(T_{h}\left(u^{i}\right)-T_{h}\left(u^{i-1}\right)\right)\right) d x \\
& \quad=\int_{\Omega_{k}^{1} \cap \Omega_{h}^{2}} a\left(x, \nabla u^{i}\right) \nabla u^{i} d x .
\end{aligned}
$$


where

$$
\begin{aligned}
& \Omega_{k}^{1}=\left\{\left|u^{i}-T_{h}\left(u^{i}+\operatorname{sgn}\left(T_{h}\left(u^{i}\right)-T_{h}\left(u^{i-1}\right)\right)\right)\right| \leq k\right\}, \\
& \Omega_{k}^{2}=\left\{\left|u^{i}-T_{h}\left(u^{i}+\operatorname{sgn}\left(T_{h}\left(u^{i}\right)-T_{h}\left(u^{i-1}\right)\right)\right)\right|>k\right\}, \\
& \Omega_{h}^{1}=\left\{\left|u^{i}\right| \leq h\right\}, \Omega_{h}^{2}=\left\{\left|u^{i}\right|>h\right\} .
\end{aligned}
$$

Then, by using (26), we deduce from the inequalities (88-90) that

$$
\int_{\Omega} a\left(x, \nabla u^{i}\right) \nabla T_{k}\left(u^{i}-T_{h}\left(u^{i}\right)+\operatorname{sgn}\left(T_{h}\left(u^{i}\right)-T_{h}\left(u^{i-1}\right)\right)\right) d x \geq 0,
$$

From (87), (88) and taking $k=1$, we get

$$
\int_{\Omega}\left(u^{i}-u^{i-1}\right) T_{1}\left(u^{i}-T_{h}\left(u^{i}\right)+\operatorname{sgn}\left(T_{h}\left(u^{i}\right)-T_{h}\left(u^{i-1}\right)\right)\right) d x \leq \tau\left(\left\|f_{i}\right\|_{1}+\left\|\beta\left(u^{i}\right)\right\|_{1}\right) .
$$

Then by applying the Lebesgue's dominated convergence theorem as $h \rightarrow 0$, we obtain

$$
\left\|u^{i}-u^{i-1}\right\|_{1} \leq \tau\left(\left\|f_{i}\right\|_{1}+\left\|\beta\left(u^{i}\right)\right\|_{1}\right) .
$$

Summing (94) from $i=1$ to $i=n$, we have

$$
\sum_{i=1}^{n}\left\|u^{i}-u^{i-1}\right\|_{1} \leq \tau \sum_{i=1}^{n}\left(\left\|f_{i}\right\|_{1}+\tau \sum_{i=1}^{n}\left\|\beta\left(u^{i}\right)\right\|_{1} .\right.
$$

Proof of (iv): We take $\varphi=0$ as test function in (37), we obtain

$$
\int_{\Omega}\left(u^{i}-u^{i-1}\right) T_{k}\left(u^{i}\right)+\tau \int_{\Omega} a\left(x, \nabla u^{i}\right) \nabla T_{k}\left(u^{i}\right)+\int_{\Omega}\left(\tau \beta\left(u^{i}\right)\right) T_{k}\left(u^{i}\right) \leq \int_{\Omega}\left(\tau f_{i}\right) T_{k}\left(u^{i}\right),
$$

by using the assumption 3.3 , we get

$$
\begin{aligned}
\tau \lambda \int_{\Omega} M\left(\left|T_{k}\left(u^{i}\right)\right|\right) d x & \leq \tau \int_{\Omega} a\left(x, \nabla T_{k}\left(u^{i}\right)\right) \nabla T_{k}\left(u^{i}\right) d x \\
& \leq \tau k\left\|f_{i}\right\|_{1}+k\left\|u^{i}-u^{i-1}\right\|_{1}+\tau k\left\|\beta\left(u^{i}\right)\right\|_{1}
\end{aligned}
$$


Summing (95) from $i=1$ to $i=n$ and applying the result (80) and (81), we obtain

$$
\sum_{i=1}^{n} \tau \int_{\Omega} M\left(\left|\nabla T_{k}\left(u^{i}\right)\right|\right) d x \leq C(f, T, k)
$$

\subsection{Existence result}

In this section we give the notion of entropy solution for the nonlinear parabolic problem (1.1) and we state the main result of this paper.

Definition 3.8 A measurable function $u: \Omega \times(0, T) \longrightarrow \mathbb{R}$ is called an entropy solution of the parabolic problem ( 1$)$ if $u \in C\left([0, T], L^{1}(\Omega)\right), T_{k}(u) \in W_{0}^{1 . x} L_{M}\left(Q_{T}\right)$ and

$$
\begin{aligned}
& \int_{\Omega} J_{k}(u-\varphi)(t) d x+\int_{Q_{T}} a(x, \nabla u) \nabla T_{k}(u-\varphi) d x d t \\
& \quad+\int_{Q_{\tau}} \beta(u) T_{k}(u-\varphi) d x d t+\int_{Q_{\tau}} \frac{\partial \varphi}{\partial t} T_{k}(u-\varphi) d x d t \\
& \quad \leq \int_{Q_{\tau}} f T_{k}(u-\varphi) d x d t+\int_{\Omega} J_{k}(u-\varphi)(0) d x
\end{aligned}
$$

for every $t \in[0, T], k>0$ and for all for all $\varphi \in L^{\infty}\left(Q_{T_{x}}\right) \cap W_{0}^{1 . x} L_{M}\left(Q_{T}\right)$, such that $\frac{\partial \varphi}{\partial t}$ belongs to $W^{-1 . x} L_{M^{*}}\left(Q_{T}\right)+L^{1}\left(Q_{T}\right)$ and that $J_{k}(x)=\int_{0}^{x} T_{k}(s) d s$.

The main result is the following:

Theorem 3.9 Under assumption (24)-(28), the nonlinear parabolic problem (1) has a unique entropy solution.

\section{Proof of theorem 3.7}

The proof is divided into 6 steps.

- Step 1: The Rothe approximate problem and a priori estimites. We introduce a piecewise linear function, called Rothe function, by

$$
\left\{\begin{array}{l}
U^{N}(0):=0 \\
\left.\left.U^{N}(t):=u^{n-1}+\left(u^{n}-u^{n-1}\right) \frac{\left(t-t^{n-1}\right)}{\tau}, \text { for all } t \in\right] t^{n-1}, t^{n}\right], n=1, \ldots, N \text { in } \Omega,
\end{array}\right.
$$


and a piecewise constant function

$$
\left\{\begin{array}{l}
\bar{U}^{N}(0):=0 \\
\left.\left.\bar{U}^{N}(t):=u^{n}, \text { for all } t \in\right] t^{n-1}, t^{n}\right], n=1, \ldots, N \text { in } \Omega
\end{array}\right.
$$

where $t^{n}:=n \tau$ and $u^{n}$ is unique solution of problem (35). We have for any $\left.t \in] t^{n-1}, t^{n}\right], n=1, \ldots, N$ that

$$
\frac{\partial U^{N}}{\partial t}=\frac{\left(u^{n}-u^{n-1}\right)}{\tau} .
$$

Remark 4.1 Using the Rothe's approximations, we can write the problem approximated of (1) as follows:

$$
\left\{\begin{array}{c}
\frac{\partial U^{N}}{\partial t}-\operatorname{div}\left(a\left(x, \nabla \bar{U}^{N}\right)\right)+\beta\left(\bar{U}^{N}\right)=f_{N} \text { in } Q_{T} \\
U^{N}=\bar{U}^{N}=0 \text { on }(0, T) \times \partial_{\Omega} \\
U^{N}(0, .)=\bar{U}^{N}(0, .)=0 \text { in } \Omega .
\end{array}\right.
$$

where $f_{N}(t, x)=f_{n}(x)$ for all $\left.\left.t \in\right] t^{n-1}, t^{n}\right], n=1, \ldots, N$.

By using the stability results, we will prove the following a priori estimates.

Proposition 4.2 Under assumption (24)-(28), there exists a constant $C_{i}(T, f)$, $(i=1, \ldots, 6)$ not depending on $N$ such that for all $N \in \mathbb{N}$, we have:

$$
\begin{aligned}
& \mid \bar{U}^{N}-U^{N} \|_{L^{1}\left(Q_{T}\right)} \leq \frac{1}{N} C_{1}(T, f) . \\
& \mid U^{N} \|_{L^{1}\left(Q_{T}\right)} \leq C_{2}(T, f) . \\
& \mid \bar{U}^{N} \|_{L^{1}\left(Q_{T}\right)} \leq C_{3}(T, f) . \\
& \left\|\frac{\partial U^{N}}{\partial t}\right\|_{L^{1}\left(Q_{T}\right)} \leq C_{4}(T, f) . \\
& \int_{Q_{T}} M\left(\left|\nabla T_{k}\left(\bar{U}^{N}\right)\right|\right) d x d t \leq k C_{5}(T, f) . \\
& \mid \beta\left(\bar{U}^{N}\right) \|_{L^{1}\left(Q_{T}\right)} \leq C_{6}(T, f),
\end{aligned}
$$

where $C_{1}(T, f)=\frac{T}{2} \sum_{n=1}^{N}\left\|u^{n}-u^{n-1}\right\|_{1}, C_{2}(T, f)=\frac{T}{2 N} \sum_{n=1}^{N}\left\|u^{n-1}\right\|_{1}+\frac{T}{2 N} \sum_{n=1}^{N}\left\|u^{n}\right\|_{1}$, 


$$
\begin{array}{r}
C_{3}(T, f)=\frac{T}{N} \sum_{n=1}^{N}\left\|u^{n}\right\|_{1}, C_{4}(T, f)=\frac{T}{N} \sum_{n=1}^{N}\left\|f_{n}\right\|_{1}+\frac{T}{N} \sum_{n=1}^{N}\left\|\beta\left(u^{n}\right)\right\|_{1}, \\
C_{5}(T, f)=\frac{T}{N} \sum_{n=1}^{N}\left\|u^{n}\right\|_{M . \Omega} \text { and } C_{6}(T, f)=\frac{T}{N} \sum_{n=1}^{N}\left\|\beta\left(u^{n}\right)\right\|_{1} .
\end{array}
$$

Proof of (i): We have

$$
\bar{U}^{N}(t)-U^{N}(t)=\left(u^{n}-u^{n-1}\right)\left(\frac{t^{n}-t}{\tau}\right) \text { and } \int_{t^{n-1}}^{t^{n}}\left(\frac{t^{n}-t}{\tau}\right) d t=\frac{\tau}{2}
$$

then, by applying (80), we get

$$
\begin{aligned}
\left\|\bar{U}^{N}-U^{N}\right\|_{L^{1}\left(Q_{T}\right)} & \leq \sum_{n=1}^{N}\left\|u^{n}-u^{n-1}\right\|_{1} \int_{t^{n-1}}^{t^{n}}\left(\frac{t^{n}-t}{\tau}\right) d t \\
& \leq \frac{\tau}{2} \sum_{n=1}^{N}\left\|u^{n}-u^{n-1}\right\|_{1} \\
& \leq \frac{T}{2 N} \sum_{n=1}^{N}\left\|u^{n}-u^{n-1}\right\|_{1} .
\end{aligned}
$$

Proof of (ii): From (97), for all $\left.t \in] t^{n-1}, t^{n}\right]$ we have

$$
\begin{aligned}
U^{N}(t) & =u^{n-1}+\left(u^{n}-u^{n-1}\right) \frac{\left(t-t^{n-1}\right)}{\tau}=u^{n-1} \frac{\left(t^{n}-t^{n-1}-t+t^{n-1}\right)}{\tau}+u^{n} \frac{\left(t-t^{n-1}\right)}{\tau} \\
& =u^{n-1} \frac{\left(t^{n}-t\right)}{\tau}+u^{n} \frac{\left(t-t^{n-1}\right)}{\tau},
\end{aligned}
$$

then

$$
\begin{aligned}
\left\|U^{N}\right\|_{L^{1}\left(Q_{T}\right)} & =\int_{0}^{T} \int_{\Omega}\left|U^{N}(t)\right| d t d x \\
& =\sum_{n=1}^{N} \int_{t^{n-1}}^{t^{n}} \int_{\Omega}\left|U^{N}(t)\right| d t d x \\
& \leq \sum_{n=1}^{N} \int_{t^{n-1}}^{t^{n}} \int_{\Omega}\left|u^{n-1}\right| \frac{\left(t^{n}-t\right)}{\tau} d t d x+\sum_{n=1}^{N} \int_{t^{n-1}}^{t^{n}} \int_{\Omega}\left|u^{n}\right| \frac{\left(t-t^{n-1}\right)}{\tau} d t d x . \\
& \leq \frac{\tau}{2} \sum_{n=1}^{N}\left\|u^{n-1}\right\|_{1}+\frac{\tau}{2} \sum_{n=1}^{N}\left\|u^{n}\right\|_{1} \\
& \leq \frac{T}{2 N} \sum_{n=1}^{N}\left\|u^{n-1}\right\|_{1}+\frac{T}{2 N} \sum_{n=1}^{N}\left\|u^{n}\right\|_{1} .
\end{aligned}
$$


Proof of (iii): From (98) and (79), we have

$$
\left.\left.\bar{U}^{N}(t):=u^{n} \text { for all } t \in\right] t^{n-1}, t^{n}\right]
$$

then

$$
\begin{aligned}
\left\|\bar{U}^{N}\right\|_{L^{1}\left(Q_{T}\right)} & =\sum_{n=1}^{N} \int_{t^{n-1}}^{t^{n}}\left\|u^{n}\right\|_{1} d t \\
& =\frac{T}{N} \sum_{n=1}^{N}\left\|u^{n}\right\|_{1} .
\end{aligned}
$$

Proof of (iv): For $t \in] t^{n-1}, t^{n}$, using (99) and (81) we get

$$
\begin{aligned}
\left\|\frac{\partial U^{N}}{\partial t}\right\|_{L^{1}\left(Q_{T}\right)} & =\sum_{n=1}^{N} \int_{t^{n-1}}^{t^{n}} \frac{1}{\tau}\left\|u^{n}-u^{n-1}\right\|_{1} d t \\
& =\sum_{n=1}^{N}\left\|u^{n}-u^{n-1}\right\|_{1} \\
& \leq \frac{T}{N} \sum_{n=1}^{N}\left\|f_{n}\right\|_{1}+\frac{T}{N} \sum_{n=1}^{N}\left\|\beta\left(u^{n}\right)\right\|_{1}
\end{aligned}
$$

Proof of (v): We have

$$
\left.\left.\bar{U}^{N}(t):=u^{n} \text { for all } t \in\right] t^{n-1}, t^{n}\right]
$$

then

$$
\begin{aligned}
\left\|T_{k}\left(\bar{U}^{N}\right)\right\|_{1 . M . \Omega} & =\sum_{n=1}^{N} \int_{t^{n-1}}^{t^{n}}\left\|u^{n}\right\|_{M . \Omega} d t \\
& =\frac{T}{N} \sum_{n=1}^{N}\left\|u^{n}\right\|_{M . \Omega} .
\end{aligned}
$$

Proof of (vi): Using the same technical as in the above, it is easy to show that

$$
\begin{aligned}
\left\|\beta\left(\bar{U}^{N}\right)\right\|_{L^{1}\left(Q_{T}\right)} & =\sum_{n=1}^{N} \int_{t^{n-1}}^{t^{n}}\left\|\beta\left(u^{n}\right)\right\|_{1} d t \\
& =\frac{T}{N} \sum_{n=1}^{N}\left\|\beta\left(u^{n}\right)\right\|_{1} .
\end{aligned}
$$


- Step 2: Convergence of the sequence $\left(\nabla T_{k}\left(\bar{U}^{N}\right)\right)_{N \in \mathbb{N}}$ in $L_{M}\left(Q_{T}\right)$. According to (102), (104) and using Aubin-Lions compactness lemma (see [19]), we deduce that the sequence $\left(U^{N}\right)_{N \in \mathbb{N}}$ is relatively compact in $L^{1}\left(Q_{T}\right)$, then there exists $u$ in $L^{1}\left(Q_{T}\right)$ such that

$$
U^{N} \rightarrow u \text { in } L^{1}\left(Q_{T}\right)
$$

Let $k>0$, by Markov inequality and (103), we deduce that

$$
\begin{aligned}
\operatorname{meas}\left(\left\{(x, t) \in Q_{T}:\left|\bar{U}^{N}\right|>k\right\}\right) & \leq \frac{1}{k}\left\|\bar{U}^{N}\right\|_{L^{1}\left(Q_{T}\right)}, \\
& \leq \frac{1}{k} C_{3}\left(T, f, u_{0}\right)
\end{aligned}
$$

so that

$\lim _{k \rightarrow \infty}\left(\right.$ meas $\left.\left\{(x, t) \in Q_{T}:\left|\bar{U}^{N}\right|>k\right\}\right)=0$ uniformly with respect to $N$.

Let $\gamma, k$ be positive real numbers and let $N, N^{\prime} \in \mathbb{N}$, we have

$$
\begin{aligned}
\operatorname{meas}\left(\left\{\left|\bar{U}^{N}-\bar{U}^{N^{\prime}}\right|>\gamma\right\}\right) \leq & \operatorname{meas}\left(\left\{\left|\bar{U}^{N}\right|>k\right\}\right)+\operatorname{meas}\left(\left\{\left|\bar{U}^{N^{\prime}}\right|>k\right\}\right) \\
& +\operatorname{meas}\left(\left\{\left|T_{k}\left(\bar{U}^{N}\right)-T_{k}\left(\bar{U}^{N^{\prime}}\right)\right|>\gamma\right\}\right)
\end{aligned}
$$

Since $T_{k}\left(\bar{U}^{N}\right)$ is bounded in $W_{0 N}^{1, x} L_{M}\left(Q_{T}\right)$ for every $k>0$, then there exists some $v_{k} \in W_{0}^{1 . x} L_{M}\left(Q_{T}\right)$, such that $T_{k}\left(\vec{U}^{N}\right) \rightarrow{ }_{N}^{k}$ weakly in $W_{0}^{1 . x} L_{M}\left(Q_{T}\right)$.

Therefore, we can assume that $T_{k}\left(\bar{U}^{N}\right)$ is a Cauchy sequence in measure in $Q_{T}$.

Let $\epsilon>0$. By (108) there exists some $k(\epsilon)>0$ such that

$$
\text { meas }\left(\left\{\left|\bar{U}^{N}-\bar{U}^{N^{\prime}}\right|>\gamma\right\}\right) \leq \epsilon \text {, for all } N, N^{\prime} \leq h_{0}(k(\epsilon), \gamma) \text {. }
$$

This proves that $\left(\bar{U}^{N}\right)_{N \in \mathbb{N}}$ is a Cauchy sequence in measure in $Q_{T}$.

On the other hand, we have

$$
\left\|\bar{U}^{N}-u\right\|_{L^{1}\left(Q_{T}\right)} \leq\left\|\bar{U}^{N}-U^{N}\right\|_{L^{1}\left(Q_{T}\right)}+\left\|U^{N}-u\right\|_{L^{1}\left(Q_{T}\right)} .
$$

Combining the estimates (79), (107) and (109), we obtain that

$$
\bar{U}^{N} \rightarrow u \quad \text { a.e. in } Q_{T}
$$

Which implies that

$$
T_{k}(u)=v_{k}
$$

Hence

$$
T_{k}\left(\bar{U}^{N}\right) \rightarrow T_{k}(u) \text { weakly in } W_{0}^{1 \cdot x} L_{M}\left(Q_{T}\right) .
$$


- In this step we prove the almost everywhere convergence of the gradients and the modular convergence of the truncations.

Lemma 4.3 Let $\left(\bar{U}^{N}\right)_{N \in \mathbb{N}}$ be the sequence defined in (98), then for any $k>0$,

$$
a\left(x, \nabla T_{k}\left(\bar{U}^{N}\right)\right) \text { is bounded in }\left(L_{M^{*}}\left(Q_{T}\right)\right)^{d} .
$$

Proof let $\varphi \in\left(E_{M}\left(Q_{T}\right)\right.$ such that $\|\varphi\|_{M} \leq 1$. In view of (25), we have

$$
\int_{Q_{\tau}}\left[a\left(x, \nabla T_{k}\left(\bar{U}^{N}\right)\right)-a(x, \varphi)\right]\left[\nabla T_{k}\left(\bar{U}^{N}\right)-\varphi\right] d x d t \geq 0 .
$$

Which gives

$$
\begin{aligned}
\int_{Q_{\tau}} a\left(x, \nabla T_{k}\left(\bar{U}^{N}\right)\right) \varphi d x d t \leq & \int_{Q_{\tau}} a\left(x, \nabla T_{k}\left(\bar{U}^{N}\right)\right) \nabla T_{k}\left(\bar{U}^{N}\right) d x d t \\
& +\int_{Q_{\tau}} a(x, \varphi)\left(\varphi-\nabla T_{k}\left(\bar{U}^{N}\right)\right) d x d t
\end{aligned}
$$

By using (24) and (105), we get

$$
\int_{Q_{\tau}} a\left(x, \nabla T_{k}\left(\bar{U}^{N}\right)\right) \nabla T_{k}\left(\bar{U}^{N}\right) d x d t \leq C_{1} k
$$

and

$$
\int_{Q_{\tau}}\left|a(x, \varphi)\left(\varphi-\nabla T_{k}\left(\bar{U}^{N}\right)\right) d x d t\right| \leq C_{2} k .
$$

Combining (113-115) and the fact ||$\varphi \|_{M} \leq 1$, we deduce that

$$
\int_{Q_{\tau}} a\left(x, \nabla T_{k}\left(\bar{U}^{N}\right)\right) \varphi d x d t \leq C_{3} k .
$$

Hence, thanks the Banach-Steinhaus Theorem, the sequence

$$
a\left(x, \nabla T_{k}\left(\bar{U}^{N}\right)\right) \text { is bounded in }\left(L_{M^{*}}\left(Q_{T}\right)\right)^{d} .
$$

Lemma 4.4 Let be the sequence defined in (98), then for any $k>0$ 


$$
\begin{gathered}
\nabla T_{k}\left(\bar{U}^{N}\right) \rightarrow \nabla T_{k}(u) \text { a.e. in } Q_{T}, \\
a\left(x, \nabla T_{k}\left(\bar{U}^{N}\right)\right) \rightarrow a\left(x, \nabla T_{k}(u)\right) \text { weakly }{ }^{*} \text { in }\left(L_{M^{*}}\left(Q_{T}\right)\right)^{d}, \\
M\left(\left|\nabla T_{k}\left(\bar{U}^{N}\right)\right|\right) \rightarrow M\left(\left|\nabla T_{k}(u)\right|\right) \text { strongly in } L^{1}\left(Q_{T}\right),
\end{gathered}
$$

as $N$ tends to $+\infty$.

Proof By using the same technics as in the proof of proposition (5.5) in paper [25], we can easily prove the desired result.

- Step 4: Convergence of the $\left(U^{N}\right)_{N \in \mathbb{N}}$ in $C\left([0, T], L^{1}(\Omega)\right)$ Let $t \in[0, T]$, using the Rothe's approximations we can write (37) in the form

$$
\begin{gathered}
\left\langle\frac{\partial U^{N}}{\partial t}, T_{k}\left(\bar{U}^{N}-\varphi\right)\right\rangle_{Q_{\tau}}+\int_{Q_{\tau}} a\left(x, \nabla \bar{U}^{N}\right) \nabla T_{k}\left(\bar{U}^{N}-\varphi\right) d x d t \\
+\int_{Q_{\tau}} \beta\left(\bar{U}^{N}\right) T_{k}\left(\bar{U}^{N}-\varphi\right) d x d t \leq \int_{Q_{\tau}} f_{N} T_{k}\left(\bar{U}^{N}-\varphi\right) d x d t,
\end{gathered}
$$

where $f_{N}(t, x)=f_{n}(x)$ for all $\left.\left.t \in\right] t^{n-1}, t^{n}\right] ; n=1 \ldots N$. Let $\left(t^{n}=n \tau\right)_{n=1}^{N},\left(t^{m} \overline{\bar{M}}^{M} m \tau^{\prime}\right)_{m=1}^{M}$ a partition of the interval $[0, T]$, let $\left(U^{N}(t), \bar{U}^{N}(t)\right)$ and $\left(U^{M}(t), \bar{U}^{M}(t)\right)$ the semi-discrete solutions defined by (97) and (98) corresponding to partitions $\left(t^{n}=n \tau\right)_{n=1}^{N},\left(t^{m}=m \tau^{\prime}\right)_{m=1}^{M}$.

Let $\underline{h}_{M} 0$, for the solution $\left(U^{N}(t), \bar{U}^{N}(t)\right)$, (resp. $\left(U^{M}(t), \bar{U}^{M}(t)\right)$, we take $\varphi=T_{h}\left(\bar{U}^{M}\right)\left(\operatorname{resp} \varphi=T_{h}\left(\bar{U}^{N}\right)\right)$ as a test function in (121), we get

$$
\begin{gathered}
\left\langle\frac{\partial U^{N}}{\partial t}, T_{k}\left(\bar{U}^{N}-T_{h}\left(\bar{U}^{M}\right)\right)\right\rangle_{Q_{\tau}}+\int_{Q_{\tau}} a\left(x, \nabla \bar{U}^{N}\right) \nabla T_{k}\left(\bar{U}^{N}-T_{h}\left(\bar{U}^{M}\right)\right) d x d t \\
+\int_{Q_{\tau}} \beta\left(\bar{U}^{N}\right) T_{k}\left(\bar{U}^{N}-T_{h}\left(\bar{U}^{M}\right)\right) d x d t \leq \int_{Q_{\tau}} f_{N} T_{k}\left(\bar{U}^{N}-T_{h}\left(\bar{U}^{M}\right)\right) d x d t
\end{gathered}
$$

and

$$
\begin{gathered}
\left\langle\frac{\partial U^{M}}{\partial t}, T_{k}\left(\bar{U}^{M}-T_{h}\left(\bar{U}^{N}\right)\right)\right\rangle_{Q_{\tau}}+\int_{Q_{\tau}} a\left(x, \nabla \bar{U}^{M}\right) \nabla T_{k}\left(\bar{U}^{M}-T_{h}\left(\bar{U}^{N}\right)\right) d x d t \\
+\int_{Q_{\tau}} \beta\left(\bar{U}^{M}\right) T_{k}\left(\bar{U}^{M}-T_{h}\left(\bar{U}^{N}\right)\right) d x d t \leq \int_{Q_{\tau}} f_{M} T_{k}\left(\bar{U}^{M}-T_{h}\left(\bar{U}^{N}\right)\right) d x d t
\end{gathered}
$$


Summing up the two inequalities $(122,123)$ and letting $h \rightarrow \infty$, we obtain

$$
\left\langle\frac{\partial\left(U^{N}-U^{M}\right)}{\partial t}, T_{k}\left(\bar{U}^{N}-\bar{U}^{M}\right)\right\rangle_{Q_{\tau}}+\lim _{h \rightarrow 0} I I_{k h}^{N, M} \leq \int_{Q_{\tau}}\left|f_{N}-f_{M}\right| d x d t,
$$

where

$$
\begin{aligned}
I I_{k h}^{N, M}= & \int_{Q_{\tau}} a\left(x, \nabla \bar{U}^{N}\right) \nabla T_{k}\left(\bar{U}^{N}-T_{h}\left(\bar{U}^{M}\right)\right) \\
& \quad+a\left(x, \nabla \bar{U}^{M}\right) \nabla T_{k}\left(\bar{U}^{M}-T_{h}\left(\bar{U}^{N}\right)\right) d x d t
\end{aligned}
$$

i.e

$$
\begin{aligned}
& \left\langle\frac{\partial\left(U^{N}-U^{M}\right)}{\partial t}, T_{k}\left(U^{N}-U^{M}\right)\right\rangle_{Q_{\tau}}+\lim _{h \rightarrow 0} I I_{k h}^{N, M} \leq \int_{Q_{\tau}}\left|f_{N}-f_{M}\right| d x d t \\
& +\left\langle\frac{\partial\left(U^{N}-U^{M}\right)}{\partial t}, T_{k}\left(U^{N}-U^{M}\right)-T_{k}\left(\bar{U}^{N}-\bar{U}^{M}\right)\right\rangle_{Q_{\tau}} .
\end{aligned}
$$

Since we have $\left\langle\frac{\partial v}{\partial t}, T_{k}(v)\right\rangle_{Q_{T}}=\frac{\partial}{\partial t} \int_{\Omega} J_{K}(v) d x$ in $L^{1}(] 0, T[)$, then the inequality (126) becomes

$$
\begin{aligned}
& \int_{\Omega} J_{k}\left(U^{N}(t)-U^{M}(t)\right) d x+\lim _{h \rightarrow 0} I I_{k h}^{N, M} \\
& \quad \leq\left|\left\langle\frac{\partial\left(U^{N}-U^{M}\right)}{\partial t}, T_{k}\left(U^{N}-U^{M}\right)-T_{k}\left(\bar{U}^{N}-\bar{U}^{M}\right)\right\rangle_{Q_{\tau}}\right|+\left|f_{N}-f_{M}\right|_{1} .
\end{aligned}
$$

On the one hand, since $T_{k}\left(\bar{U}^{N}-\varphi\right) \rightarrow T_{k}(U-\varphi)$ weakly* in $L^{\infty}\left(Q_{T}\right)$ as $N \rightarrow \infty$ and $\left\|\frac{\partial\left(U^{N}-U^{M}\right)}{\partial t}\right\|_{L^{1}\left(Q_{T}\right)} \leq C(T, f)$, we obtain

$$
\lim _{N, M \rightarrow \infty}\left|\left\langle\frac{\partial\left(U^{N}-U^{M}\right)}{\partial t}, T_{k}\left(U^{N}-U^{M}\right)-T_{k}\left(\bar{U}^{N}-\bar{U}^{M}\right)\right\rangle_{Q_{\tau}}\right|=0
$$

On the other hand, we have $\lim _{N, M \rightarrow \infty}\left\|f_{N}-f_{M}\right\|_{1}=0$.

Using the same technique used in proof of the uniqueness theorem (3.6), we prove that $\lim _{N, M \rightarrow \infty h \rightarrow 0} \lim _{k h} I, M \geq 0$.

So the passage to the limit with $N, M \rightarrow \infty$ implies

$$
\lim _{N, M \rightarrow \infty} \int_{\Omega} J_{k}\left(U^{N}(t)-U^{M}(t)\right) d x=0 .
$$

We deduce then that (see for instance, the proof of Theorem 1.1 of [30]), 


$$
\lim _{N, M \rightarrow \infty} \int_{\Omega}\left|U^{N}(t)-U^{M}(t)\right| d x=0,
$$

and thus the sequence $\left(U^{N}\right)$ is Cauchy in $C\left([0, T], L^{1}(\Omega)\right)$.

Since $U^{N} \rightarrow u$ a.e, then $U^{N} \rightarrow u$ in $C\left([0, T], L^{1}(\Omega)\right)$.

- Step 5: Passage to the limit. It remains to show that the function $u$ is the entropy solution of problem ( 1$)$. Let $v \in W^{1, x} L_{M}\left(Q_{T}\right) \cap L^{\infty}$ such that $\frac{\partial v}{\partial t}$ belongs to $W^{-1, x} L_{M^{*}}\left(Q_{T}\right)+L^{1}\left(Q_{T}\right)$. By Lemma 5 and Theorem 3 in [12], there exists a prolongation $\bar{v}=v$ on $Q_{T}, \bar{v} \in W^{1, x} L_{M}(\Omega \times \mathbb{R}) \cap L^{1}(\Omega \times \mathbb{R}) \cap L^{\infty}(\Omega \times \mathbb{R})$, and

$$
\frac{\partial v}{\partial t} \in W^{-1, x} L_{M^{*}}(\Omega \times \mathbb{R})+L^{1}(\Omega \times \mathbb{R}) .
$$

There exists also a sequence $\left(\omega_{j}\right) \subset \mathcal{D}(\Omega \times \mathbb{R})$ such that

$$
\begin{aligned}
& \omega_{j} \rightarrow \bar{v} \text { in } W_{0}^{1, x} L_{M}(\Omega \times \mathbb{R}) \text {, and } \\
& \frac{\partial \omega_{j}}{\partial t} \rightarrow \frac{\partial \bar{v}}{\partial t} \text { in } W^{-1, x} L_{M^{*}}(\Omega \times \mathbb{R})+L^{1}(\Omega \times \mathbb{R}) .
\end{aligned}
$$

Now, let take $T_{k}\left(\bar{U}^{N}-\omega_{j}\right)_{\chi_{(0, t)}}$ as a test function in (121), thus for every $t \in[0, T]$, we get

$$
\begin{gathered}
\left\langle\frac{\partial U^{N}}{\partial t}, T_{k}\left(\bar{U}^{N}-\omega_{j}\right)\right\rangle_{Q_{\tau}}+\int_{Q_{\tau}} a\left(x, \nabla T_{\bar{k}}\left(\bar{U}^{N}\right)\right) \nabla T_{k}\left(\bar{U}^{N}-\omega_{j}\right) d x d t \\
+\int_{Q_{\tau}} \beta\left(\bar{U}^{N}\right) T_{k}\left(\bar{U}^{N}-\omega_{j}\right) d x d t=\int_{Q_{\tau}} f_{N} T_{k}\left(\bar{U}^{N}-\omega_{j}\right) d x d t
\end{gathered}
$$

where $f_{N}(t, x)=f_{n}(x)$ for all $\left.\left.t \in\right] t^{n-1}, t^{n}\right], n=1 \ldots N$, and $\bar{k}=k+C\left\|\omega_{j}\right\|_{\infty, Q}$. Which implies,

$$
\begin{aligned}
& \left\langle\frac{\partial U^{N}}{\partial t}, T_{k}\left(U^{N}-\omega_{j}\right)\right\rangle_{Q_{\tau}}+\left\langle\frac{\partial U^{N}}{\partial t}, T_{k}\left(\bar{U}^{N}-\omega_{j}\right)-T_{k}\left(U^{N}-\omega_{j}\right)\right\rangle_{Q_{\tau}} \\
& \quad+\int_{Q_{\tau}} a\left(x, \nabla T_{\bar{k}}\left(\bar{U}^{N}\right)\right) \nabla T_{k}\left(\bar{U}^{N}-\omega_{j}\right) d x d t+\int_{Q_{\tau}} \beta\left(\bar{U}^{N}\right) T_{k}\left(\bar{U}^{N}-\omega_{j}\right) d x d t \\
& \quad \leq \int_{Q_{\tau}} f_{N} T_{k}\left(\bar{U}^{N}-\omega_{j}\right) d x d t .
\end{aligned}
$$

Since

$$
\left\langle\frac{\partial U^{N}}{\partial t}, T_{k}\left(U^{N}-\omega_{j}\right)\right\rangle_{Q_{\tau}}=\left\langle\frac{\partial\left(U^{N}-\omega_{j}\right)}{\partial t}, T_{k}\left(U^{N}-\omega_{j}\right)\right\rangle_{Q_{\tau}}+\left\langle\frac{\partial \omega_{j}}{\partial t}, T_{k}\left(U^{N}-\omega_{j}\right)\right\rangle_{Q_{\tau}}
$$


and

$$
\left\langle\frac{\partial\left(U^{N}-\omega_{j}\right)}{\partial t}, T_{k}\left(U^{N}-\omega_{j}\right)\right\rangle_{Q_{\tau}}=\int_{\Omega} J_{k}\left(U^{N}(t)-\omega_{j}(t)\right) d x-\int_{\Omega} J_{k}\left(U^{N}(0)-\omega_{j}(0)\right) d x .
$$

Then, we deduce that

$$
\begin{gathered}
\left\langle\frac{\partial U^{N}}{\partial t}, T_{k}\left(U^{N}-\omega_{j}\right)\right\rangle_{Q_{\tau}}=\int_{\Omega} J_{k}\left(U^{N}(t)-\omega_{j}(t)\right) d x-\int_{\Omega} J_{k}\left(U^{N}(0)-\omega_{j}(0)\right) d x \\
+\left\langle\frac{\partial \omega_{j}}{\partial t}, T_{k}\left(U^{N}-\omega_{j}\right)\right\rangle_{Q_{\tau}} .
\end{gathered}
$$

We have $\left(U^{N}\right)_{N \in \mathbb{N}}$ converges to $u$ in $C\left([0, T], L^{1}(\Omega)\right)$, then $U^{N}(t) \rightarrow u(t)$ in $L^{1}(\Omega)$ for all $t \in[0, T]$. Moreover, since $\left|J_{k}\left(U^{N}(t)-\omega_{j}(t)\right)\right| \leq k\left|U^{N}(t)\right|+k\left|\omega_{j}(t)\right|$, then we have by using Lebesgue theorem

$$
\int_{\Omega} J_{k}\left(U^{N}(t)-\omega_{j}(t)\right) d x \rightarrow \int_{\Omega} J_{k}\left(u(t)-\omega_{j}(t)\right) d x \text { as } N \rightarrow \infty .
$$

Therefore we can pass to the limit in (133), we obtain

$$
\begin{aligned}
\lim _{N \rightarrow \infty}\left\langle\frac{\partial U^{N}}{\partial t}, T_{k}\left(U^{N}-\omega_{j}\right)\right\rangle_{Q_{\tau}}= & \int_{\Omega} J_{k}\left(u-\omega_{j}\right) d x-\int_{\Omega} J_{k}\left(u_{0}-\omega_{j}(0)\right) d x \\
& +\left\langle\frac{\partial \omega_{j}}{\partial s}, T_{k}\left(u-\omega_{j}\right)\right\rangle_{Q_{\tau}}
\end{aligned}
$$

For the second term of the (131), since $T_{k}\left(\bar{U}^{N}-\omega_{j}\right) \rightarrow T_{k}\left(u-\omega_{j}\right)$ weakly* in $L^{\infty}\left(Q_{T}\right)$ as $N \rightarrow \infty$ and $\left\|\frac{\partial U^{N}}{\partial t}\right\|_{L^{1}\left(Q_{T}\right)} \leq C(T)$, then

$$
\lim _{N \rightarrow \infty}\left|\left\langle\frac{\partial U^{N}}{\partial t}, T_{k}\left(\bar{U}^{N}-\omega_{j}\right)-T_{k}\left(U^{N}-\omega_{j}\right)\right\rangle_{Q_{T}}\right|=0
$$

The third term of (131) reads as

$$
\begin{aligned}
\int_{Q_{\tau}} a\left(x, \nabla T_{\bar{k}}\left(\bar{U}^{N}\right)\right) \nabla T_{k}\left(\bar{U}^{N}-\omega_{j}\right) d x d t= & \int_{Q_{1 . T}} a\left(x, \nabla T_{\bar{k}}\left(\bar{U}^{N}\right)\right) \nabla\left(T_{\bar{k}}(\bar{U})^{N}\right) d x d t \\
& -\int_{Q_{1 . T}} a\left(x, \nabla T_{\bar{k}}\left(\bar{U}^{N}\right)\right) \nabla \omega_{j} d x d t
\end{aligned}
$$

where $Q_{1 . T}=Q_{T} \cap\left\{\left|\bar{U}^{N}-\omega_{j}\right| \leq k\right\}$.

Thanks to lemma (4.4), and by using Fatou's lemma, we deduce that 


$$
\int_{Q_{1 . T}} a\left(x, \nabla T_{\bar{k}}\left(\bar{U}^{N}\right)\right) \nabla T_{\bar{k}}\left(\bar{U}^{N}\right) d x d t \rightarrow \int_{Q_{1 . T}} a\left(x, \nabla T_{\bar{k}}(u)\right) \nabla T_{\bar{k}}(u) d x d t
$$

and

$$
\int_{Q_{1 . T}} a\left(x, \nabla T_{\bar{k}}\left(\bar{U}^{N}\right)\right) \nabla \omega_{j} d x d t \rightarrow \int_{Q_{1 . T}} a\left(x, \nabla T_{\bar{k}}(u)\right) \nabla \omega_{j} d x d t
$$

Hence

$$
\int_{Q_{\tau}} a\left(x, \nabla \bar{U}^{N}\right) \nabla T_{k}\left(\bar{U}^{N}-\omega_{j}\right) d x d t \rightarrow \int_{Q_{\tau}} a(x, \nabla u) \nabla T_{k}\left(u-\omega_{j}\right) d x d t .
$$

Since $T_{k}\left(\bar{U}^{N}-\omega_{j}\right) \rightarrow T_{k}\left(u-\omega_{j}\right)$ weakly* in $L^{\infty}\left(Q_{T}\right)$ as $N \rightarrow \infty$, we have

$$
\int_{Q_{\tau}} f_{N} T_{k}\left(\bar{U}^{N}-\omega_{j}\right) d x d t \rightarrow \int_{Q_{\tau}} f T_{k}\left(u-\omega_{j}\right) d x d t .
$$

And thus, using the above results $(132,136,140,141)$ and the continuity of $\beta$, we deduce that

$$
\begin{aligned}
& \int_{\Omega} J_{k}\left(u-\omega_{j}\right) d x-\int_{\Omega} J_{k}\left(u_{0}-\omega_{j}(0)\right) d x+\left\langle\frac{\partial \omega_{j}}{\partial t}, T_{k}\left(u-\omega_{j}\right)\right\rangle_{Q_{\tau}} \\
& \quad+\int_{Q_{\tau}} a(x, \nabla u) \nabla T_{k}\left(u-\omega_{j}\right) d x d t+\int_{Q_{\tau}} \beta(u) T_{k}\left(u-\omega_{j}\right) d x d t \\
& \quad \leq \int_{Q_{\tau}} f T_{k}\left(u-\omega_{j}\right) d x d t .
\end{aligned}
$$

By (130), as $j$ tends to $+\infty$ we have

$$
\left\langle\frac{\partial \omega_{j}}{\partial t}, T_{k}\left(u-\omega_{j}\right)\right\rangle_{Q_{\tau}} \rightarrow\left\langle\frac{\partial v}{\partial t}, T_{k}(u-v)\right\rangle_{Q_{\tau}}
$$

Moreover, for every $t \in[0, T]$, we have $\left\|\omega_{j}-v(t)\right\|_{L^{1}(\Omega)} \rightarrow 0$ as $j \rightarrow+\infty$. Therefore, we pass now to the limit as $j \rightarrow+\infty$ in (142), we get

$$
\begin{aligned}
& \int_{\Omega} J_{k}(u-v) d x-\int_{\Omega} J_{k}\left(u_{0}-v(0)\right) d x+\left\langle\frac{\partial v}{\partial t}, T_{k}(u-v)\right\rangle_{Q_{\tau}} \\
& \quad+\int_{Q_{\tau}} a(x, \nabla u) \nabla T_{k}(u-v) d x d t+\int_{Q_{\tau}} \beta(u) T_{k}(u-v) d x d t \\
& \leq \int_{Q_{\tau}} f T_{k}(u-v) d x d t
\end{aligned}
$$


then $u$ is an entropy solution of the nonlinear parabolic problem (1), and this completes the proof of existence of the entropy solution.

- Let $v$ be another entropy solution of nonlinear parabolic problem (1). We take $\varphi=T_{h}\left(U^{N}\right)$ as test function. Passing to the limit with $h \rightarrow \infty$, we obtain

$$
\begin{gathered}
\int_{Q_{\tau}} a(x, \nabla v) \nabla T_{k}\left(v-T_{h}\left(U^{N}\right)\right) d x d t+\int_{Q_{\tau}} \beta(v) T_{k}\left(v-T_{h}\left(U^{N}\right)\right) d x d t \\
\leq \int_{Q_{\tau}} f T_{k}\left(v-T_{h}\left(U^{N}\right)\right) d x d t+\int_{\Omega} J_{k}(v(0)-\varphi(0)) d x \\
\quad-\int_{\Omega} J_{k}(v(t)-\varphi(t)) d x-\left\langle\frac{\partial \varphi}{\partial t}, T_{k}\left(v-T_{h}\left(U^{N}\right)\right)\right\rangle_{Q_{\tau}}
\end{gathered}
$$

then

$$
\begin{gathered}
\int_{\Omega} J_{k}\left(v(t)-U^{N}\right) d x+\left\langle\frac{\partial U^{N}}{\partial t}, T_{k}\left(v-U^{N}\right)\right\rangle_{Q_{\tau}}+\lim _{h \rightarrow \infty} I I_{1}^{N}(k, h) \\
+\int_{Q_{\tau}} \beta(v) T_{k}\left(v-U^{N}\right) d x d t \leq \int_{Q_{\tau}} f T_{k}\left(v-U^{N}\right) d x d t,
\end{gathered}
$$

where

$$
I_{1}^{N}(k, h)=\int_{Q_{\tau}} a(x, \nabla v) \nabla T_{k}\left(v-T_{h}\left(U^{N}\right)\right) d x d t .
$$

On the other hand, taking $\varphi=T_{h}(v)$ as test function in (121), and by letting $h$ to $\infty$, we obtain

$$
\begin{gathered}
\left\langle\frac{\partial U^{N}}{\partial t}, T_{k}\left(\bar{U}^{N}-T_{h}(v)\right)\right\rangle_{Q_{\tau}}+\int_{Q_{\tau}} a\left(x, \nabla \bar{U}^{N}\right) \nabla T_{k}\left(\bar{U}^{N}-T_{h}(v)\right) d x d t \\
+\int_{Q_{\tau}} \beta\left(\bar{U}^{N}\right) T_{k}\left(\bar{U}^{N}-T_{h}(v)\right) d x d t \leq \int_{Q_{\tau}} f_{N} T_{k}\left(\bar{U}^{N}-T_{h}(v)\right) d x d t
\end{gathered}
$$

and

$$
\begin{aligned}
& \left\langle\frac{\partial U^{N}}{\partial t}, T_{k}\left(\bar{U}^{N}-v\right)\right\rangle_{Q_{\tau}}+\lim _{h \rightarrow \infty} I I_{2}^{N}(k, h)+\int_{Q_{\tau}} \beta\left(\bar{U}^{N}\right) T_{k}\left(\bar{U}^{N}-v\right) d x d t \\
& \quad \leq \int_{Q_{\tau}} f_{N} T_{k}\left(\bar{U}^{N}-v\right) d x d t
\end{aligned}
$$


where

$$
I I_{2}^{N}(k, h)=\int_{Q_{\tau}} a\left(x, \nabla \bar{U}^{N}\right) \nabla T_{k}\left(\bar{U}^{N}-v\right) d x d t .
$$

Summing up the two inequalities ( 145,147$)$, we obtain

$$
\begin{aligned}
& \int_{\Omega} J_{k}\left(v(t)-U^{N}(t)\right) d x+\left\langle\frac{\partial U^{N}}{\partial t}, T_{k}\left(v-U^{N}\right)+T_{k}\left(\bar{U}^{N}-v\right)\right\rangle_{Q_{\tau}} \\
& \quad+\lim _{h \rightarrow \infty} I I^{N}(k, h)+\int_{Q_{\tau}} \beta(v) T_{k}\left(v-U^{N}\right)+\beta\left(\bar{U}^{N}\right) T_{k}\left(\bar{U}^{N}-v\right) d x d t \\
& \quad \leq \int_{Q_{\tau}} f_{N} T_{k}\left(v-U^{N}\right)+f_{N} T_{k}\left(\bar{U}^{N}-v\right) d x d t
\end{aligned}
$$

where $I I^{N}(k, h)=I I_{1}^{N}(k, h)+I I_{2}^{N}(k, h)$. By using the results of convergence and the hypothesis (28), we get

$$
\lim _{N \rightarrow \infty} \lim _{h \rightarrow \infty}\left\langle\frac{\partial U^{N}}{\partial t}, T_{k}\left(v-U^{N}\right)+T_{k}\left(\bar{U}^{N}-v\right)\right\rangle_{Q_{\tau}}=0,
$$

and

$$
\lim _{N \rightarrow \infty h \rightarrow \infty} \lim _{Q_{\tau}} f_{N} T_{k}\left(v-U^{N}\right)+f_{N} T_{k}\left(\bar{U}^{N}-v\right) d x d t=0 .
$$

Combining $(148,150)$, we deduce that

$$
\int_{\Omega} J_{k}(v(t)-u(t)) d x+\lim _{N \rightarrow \infty} \lim _{h \rightarrow \infty} I I^{N}(k, h) \leq 0 .
$$

By applying the same technique used in the proof of the uniqueness theorem (3.6), we prove that

$$
\lim _{N \rightarrow \infty} \lim _{h \rightarrow \infty} I I^{N}(k, h) \geq 0
$$

then

$$
\int_{\Omega} J_{k}(v(t)-u(t)) d x \leq 0
$$

since $\lim _{k \rightarrow 0} \frac{J_{k}(s)}{k}=|s|$, then $\lim _{k \rightarrow 0} \frac{J_{k}(v(t)-u(t))}{k}=|v(t)-u(t)|$.

Therefore, according to Fatou's lemma, we obtain

$$
\|v(t)-u(t)\|_{1} \leq 0 \forall t \in[0, T] .
$$

Hence the uniqueness of the entropy solution of the problem (1), and the proof of theorem (3.9) is complete. 


\section{Compliance with ethical standards}

Conflict of interest The authors declare no conflict of interest, and the founding sponsors had no role in the design of the study, in the writing of the manuscript, and in the decision to publish the results.

Open Access This article is distributed under the terms of the Creative Commons Attribution 4.0 International License (http://creativecommons.org/licenses/by/4.0/), which permits unrestricted use, distribution, and reproduction in any medium, provided you give appropriate credit to the original author(s) and the source, provide a link to the Creative Commons license, and indicate if changes were made.

\section{References}

1. Adams, R.: Sobolev Spaces. Academic Press, New York (1975)

2. Aharouch, L., Azroul, E., Rhoudaf, M.: Existence of solutions for unilateral problems in $L^{1}$ involving lower order terms in divergence form in Orlicz spaces. J. Appl. Anal. 13, 151-181 (2007)

3. Azroul, E., Redwane, H., Rhoudaf, M.: Existence of a renormalized solution for a class of nonlinear parabolic equations in Orlicz Spaces. Port. Math. 66(1), 29-63 (2009)

4. Bnilan, P., Boccardo, L., Gallouët, T., Gariepy, R., Pierre, M., Vasquez, J.-L.: An $L^{1}$-theory of existence and uniqueness of solutions of nonlinear elliptic equations. Ann. Scuola Norm. Sup. Pisa 22, 241-273 (1995)

5. Boccardo, L., Giachetti, D., Diaz, J.-I., Murat, F.: Existence and regularity of renormalized solutions for some elliptic problems involving derivation of nonlinear terms. J. Difer. Equ. 106, 215-237 (1993)

6. DiPerna, R.-J., Lions, P.-L.: On the Cauchy problem for Boltzmann equations: global existence and weak stability. Ann. Math. 130, 321-366 (1989)

7. Droniou, J., Prignet, Alain: Equivalence between entropy and renormalized solutions for parabolic equations with smooth measure data. Nonlinear Differ. Equ. Appl. 14(12), 181205 (2007)

8. Emmrich, E., Wróblewska-Kaminska, A.: Convergence of a full discretization of quasi-linear parabolic equations in isotropic and anisotropic Orlicz spaces. SIAM J. Numer. Anal. 51, 1163-1184 (2013)

9. El Hachimi, A., Jamea, A.: Nonlinear parabolic problems with Neumann-type boundary conditions and $L^{1}$-data. Electron. J. Qual. Theory Differ. Equ. 27, 1-22 (2007)

10. Elmahi, A., Meskine, D.: Non-linear elliptic problems having natural growth and $L^{1}$ data in Orlicz spaces. Ann. Mat. 184, 161-184 (2005)

11. Elmahi, A., Meskine, D.: Strongly nonlinear parabolic equations with natural growth terms and $L^{1}$ data in Orlicz spaces, Portugaliae Mathematica. Nova 62, 143-183 (2005)

12. Elmahi, A., Meskine, D.: Strongly nonlinear parabolic equations with natural growth terms in Orlicz spaces, Nonlinear Analisis. Theory Methods Appl. 60(1), 1-35 (2005)

13. Gossez, J.P.: Nonlinear elliptic boundary value problems for equations with rapidly (or slowly) increasing coefficients. Trans. Am. Math. Soc. 190, 163-205 (1974)

14. Gossez, J.P.: Some approximation properties in Orlicz-Sobolev. Studia Math. 74, 17-24 (1982)

15. Gossez, J.P., Mustonen, V.: Variational inequalities in Orlicz-Sobolev spaces. Nonlinear Anal. 11, 379-492 (1987)

16. Gwiazda, P., Skrzypczak, I., Zatorska-Goldstein, A.: Existence of renormalized solutions to elliptic equation in Musielak-Orlicz space. J. Differ. Equ. 264(1), 341-377 (2018)

17. Gwiazda, P., Swierczewska-Gwiazda, A.: Parabolic equations in anisotropic Orlicz spaces with general N-functions. Special Birkhaeuser volume Parabolic problems. The Herbert Amann Festschrift. Prog. Nonlinear Differ. Equ. Appl. 60, 301311 (2010)

18. Gwiazda, P., Wittbold, P., Wrblewska-Kamiska, A., Zimmermann, A.: Renormalized solutions to nonlinear parabolic problems in generalized MusielakOrlicz spaces. Nonlinear Anal. Theory Methods Appl. 129, 1-36 (2015)

19. Jaques, S.: Compact sets in the space $L^{p}(0 ; T ; B)$. Ann. Math. Pura Appl. 146, 65-96 (1987)

20. Kac̀ur, J, : Method of Rothe in Evolution Equations. Institute of Applied Mathematics, Bratislava (1986) 
21. Kouraichi, C., Siai, A.: Equivalence between entropy and renormalized solutions for parabolic equations. Indagat. Math. 26, 679-696 (2015)

22. Krasnoselskii, M., Rutickii, Ya.: Convex Functions and Orlicz Spaces. Noordhoff, Groningen (1969)

23. Lions, J.-L.: Quelques méthodes de résolution des problèmes aux limites non linéaire. Dunod et Gauthier-Villars, Paris (1969)

24. Lions, P.-L.: Mathematical Topics in Fluid Mechanics, Incompressible models. Oxford University Press, Oxford (1996)

25. Mabdaoui, M., Moussa, H., Rhoudaf, M.: Entropy solutions for a nonlinear parabolic problems with lower order term in Orlicz spaces. Anal. Math. Phys. 7, 1 (2016)

26. Moussa, H., Rhoudaf, M.: Study of some non-linear eliptic problems with no continuous lower order terms in Orlicz spaces. Mediterranean J. Math. 13(6), 48674899 (2016)

27. Murat, F.: Soluciones renormalizadas de EDP elipticas non lineales. Cours à l'Université de Séville, Paris (1993)

28. Prignet, A.: Existence and uniqueness of entropy solutions of parabolic problems with $L^{1}$ data. Nonlinear Anal. TMA 28, 1943-1954 (1997)

29. Perona, P., Malik, J.: Scale-space and edge detection using anisotropic diffusion. IEEE Trans. Pattern Anal. Mach. Intell. 12, 629-639 (1990)

30. Porretta, A.: Nonlinear equations with natural growth terms and measure data, Electronic J. Diff. Equ. In: Conference 09, pp 181-202 (2002)

31. Rajagopal, K.R., Ružička, M.: Mathematical modeling of electrorheological materials. Contin. Mech. Thermodyn. 13, 59-78 (2001)

32. Rektorys, K.: The Method of Discretization in Time and Partial Differential Equations, vol. 4 of Mathematics and Its Applications (East European Series), D. Reidel, Dordrecht, The Netherlands. View at MathSciNet (1982)

33. Ružička, M., : Electrorheological fluids. modeling and mathematical theory, lecture notes in mathematics. Springer, Berlin (2000)

Publisher's Note Springer Nature remains neutral with regard to jurisdictional claims in published maps and institutional affiliations. 